\title{
Research on nonlinear vibration control of laminated cylindrical shells with discontinuous piezoelectric layer
}

Chaofeng Li ( $\nabla$ chfli@mail.neu.edu.cn )

Northeastern University https://orcid.org/0000-0001-6335-5608

\section{Peiyong Li}

Northeastern University

\section{Xueyang Miao}

Northeastern University

\section{Original Research}

Keywords: Geometrically nonlinear, piezoelectric, vibration control, elastic boundary condition

Posted Date: February 8th, 2021

DOl: https://doi.org/10.21203/rs.3.rs-192617/v1

License: (c) (1) This work is licensed under a Creative Commons Attribution 4.0 International License.

Read Full License 


\title{
Research on nonlinear vibration control of laminated cylindrical shells with discontinuous piezoelectric layer
}

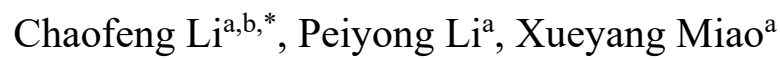 \\ a. School of Mechanical Engineering and Automation, Northeastern University, 110819, Shenyang, \\ China \\ b. Key Laboratory of Vibration and Control of Aero-Propulsion Systems Ministry of Education of \\ China, Northeastern University, Shenyang, Liaoning, China, 110819
}

\begin{abstract}
In this paper, the nonlinear vibration control of the piezoelectric laminated cylindrical shell with point supported elastic boundary condition is analyzed, in which the geometric nonlinearity is considered by the first-order shear nonlinear shell theory. In the model, different boundary conditions are simulated by introducing a series of artificial springs. The elastic-electrically coupled differential equations of piezoelectric laminated cylindrical shells are obtained based on the Chebyshev polynomials and Lagrange equation, and decoupled by using the negative velocity feedback adjustment. Later, the Incremental Harmonic Balance Method (IHBM) is deduced, and the frequencyamplitude response of the piezoelectric laminated cylindrical shell is obtained by IHBM. Finally, the influence of the constant gain, size and position of the piezoelectric layer on frequency-amplitude response are investigated. The results show that the position, size and constant gain of the piezoelectric layer have a significant influence on its nonlinear vibration control.
\end{abstract}

Keyword: Geometrically nonlinear, piezoelectric, vibration control, elastic boundary condition

\section{Introduction}

In recent years, with the development of piezoelectric materials, the piezoelectric films (PVDF) have been widely used in the fields of machinery, environmental testing, and medicine because of their functions of vibration detection, vibration control, and energy collection. The thin-walled cylindrical shell structure is an essential structure in aerospace and other fields. Because the environment of the cylindrical shell is mostly complicated, the vibration control problem has always

${ }^{*}$ Corresponding author. Tel.: +86 13514215459

E-mail address: chfli@mail.neu.edu.cn (C. Li). 
been the focus and hotspot of scholars.

Since the advent of piezoelectric intelligent mechanisms, the study of the vibration characteristics of piezoelectric laminated cylindrical shells has continued to attract scholar's attention $[1,2]$. The modeling of piezoelectric laminated shells mainly focuses on the Finite Element Method and Hamilton's principle. Kerur et al. [3] presented a finite element model using a composite material (AFC) as the piezoelectric actuator and a piezoelectric film (PVDF) as the piezoelectric sensor, and used the negative velocity feedback control algorithm to control the dynamic response of the laminated composite plate. Parashar and Kumar [4] used the Rayleigh-Ritz method to study the vibration behavior of the piezoelectric shells, and the results showed that the Rayleigh-Ritz method had a very high convergence rate and can be calculated with relatively less efforts. Sheng and Wang[5] analyzed the dynamics characteristic of functionally graded material(FGM) cylindrical shells with a surface-bonded PZT piezoelectric layer by using the first-order shear deformation theory (FSDT) and Hamilton's principle, in which the thermal and moving loads are considered in the model. Qin et al. [6] used Sanders shell theory combined with the artificial spring technique to study the free vibration of cylindrical shells under arbitrary boundary conditions, and compared the accuracy, convergence rate and computational efficiency of three different admissible displacement functions, namely the modified Fourier series, the Orthogonal polynomials, and the Chebyshev polynomials, and the results showed that Chebyshev polynomials obtained higher computational efficiency. Further, using Chebyshev polynomials as admissible displacement functions and FSDT, Qin et al.[7] proposed a general method to study the free vibration of rotating functionally graded carbon nanotube reinforced composite (FG-CNTRC) cylindrical shells with arbitrary boundary conditions. The Lagrange equation is simple to use and has a small dimension, which can well handle the dynamic modeling of piezoelectric laminated cylindrical shells. Li et al. [8]presented a model of discontinuous piezoelectric 
laminated shell with point supported elastic boundary conditions based on the first-order shear shell theory, the Chebyshev polynomials and the Lagrange equation. Then, the location of piezoelectric layer was optimized by using the Multi-Objective Particle Swarm Optimization algorithm. In practical applications, the thin-walled cylindrical shell structures, such as the aero-engine casing, are subjected to complex loads in a relatively complex environment. In this case, the geometric nonlinearity of cylindrical shell structures is inevitable. Therefore, it is of great significance to study the nonlinear amplitude-frequency response of cylindrical shells. With the deepening of the research on piezoelectric laminated cylindrical shells, Many researchers have applied piezoelectric materials to the vibration control of thin-walled cylindrical shell structures. Amabili [9] considered the axial symmetry of laminated cylindrical shells and calculated the nonlinear forced vibration response for the first time, and compared the classical Novozhilov theory, the usual version of higher-order shear deformation theory, and the higher-order shear deformation theory recently developed by Amabili and Reddy. The results show that the Amabili-Reddy and Novozhilov theories have good results for laminated thin shells. For thick shells, the Amabili-Reddy theory should be used in order to have accurate results. Jansen[10] studied the effects of static loads and imperfections on the nonlinear vibration characteristics of cylindrical shells based on the Donnell-type governing equations. Przekop electro-thermal-pneumatic-mechanical coupling load. Then, the effects of piezoelectric layer 

laminated cylindrical shells with thin piezoelectric layers in which the inner layer is a sensing layer, the functionally graded layer, and the outer layer is the active layer, and uses the Von Kármán nonlinear theory, the Hamilton's principle, the FSDT and the multiterm Galerkin method to analyze the active vibration control of smart FG laminated cylindrical shells. Shen and Yang [15] used the higher order shear deformation shell theory with a von Kármán-type of kinematic nonlinearity to study the small and large amplitude flexural vibrations of anisotropic shear deformable laminated cylindrical shells with piezoelectric fiber reinforced composite (PFRC) actuators in thermal environments. Zhang[16] used various geometrically nonlinear shell theories based on the FSDT, including refined von Kármán nonlinear shell theory(RVK5), moderate rotation shell theory(MRT5), fully geometrically nonlinear shell theory with moderate rotations(LRT5) and fully geometrically nonlinear shell theory with large rotations(LRT56), the results showed that the LRT56 can get more accurate results when structures undergo large rotations, and by adopting appropriate feedback actuation voltages, shape and vibration control can be accomplished pretty well in the structures undergoing large deformations and large rotations. Yue et al. [17] established an experimental platform for adaptive modal control of precision paraboloidal shell, conducted an experimental study on the active modal vibration control of a flexible paraboloidal shell with free boundary conditions, and used the positive position feedback algorithm to control the first order mode as well as the first and second order coupled mode of the paraboloidal shell. Ninh et al.[18] established the model of functionally graded carbon nanotube reinforced composite(FG-CNTRC) cylindrical shells with the piezoelectric actuators surrounded by an elastic medium and used the classical shell theory with geometrical nonlinearity to investigate its electro-thermo-mechanical vibration.

In recent years, many scholars have proposed discontinuous elastic boundaries and nonlinear 
flexible boundaries to replace traditional classical boundaries. Chen et al. [21] extended the improved

2 Fourier series method to the free vibration analysis of cylindrical shells, and studied the free vibration

3 of cylindrical shells with arbitrary and non-uniformly restrained boundary conditions. Xie et al. [22] used the wave propagation method to study the free vibration and forced vibration of a non-uniform constrained cylindrical shell. Li et al. $[19,20]$ studied the geometrically nonlinear vibration of noncontinuous elastic-supported laminated composite cylindrical thin shells by extending a new arcssupported and points-supported shell model. Tang et al. $[23,24]$ considered the three contact states of bolted connection, including stick, slip and separation, and established a piecewise linear boundary analytical model of bolted cylindrical shells, and studied its dynamic characteristics. Li et al.[25] presented a model of laminated composited cylindrical shells with arc supported and point supported elastic boundary condition based on Donnell's shell theory, the Chebyshev polynomials and the Lagrange equation.

According to the above literature research, the research on the dynamic characteristic of laminated shells with piezoelectric materials has attracted much attention. In addition, the vibration control of cylindrical shells by piezoelectric materials has been confirmed in some researches, but the influence of piezoelectric materials on the nonlinear vibration of cylindrical shell remains to be studied. Due to the fabrication process and cost of the piezoelectric material, it is very difficult to completely cover the piezoelectric layer on the surface of the cylindrical shell. Therefore, we innovatively proposed the nonlinear dynamic model of laminated cylindrical shells with discontinuous piezoelectric layers to study the influence of piezoelectric layers of different sizes and positions on the frequency-amplitude response. The Chebyshev polynomial, Lagrange equation, first-order shear nonlinear shell theory and Negative speed feedback strategy are used to obtain the differential equation of decoupled motion. Afterward, the IHBM is used to solve the differential equation of motion, and the effects of constant 


\begin{tabular}{|c|c|}
\hline \multicolumn{2}{|l|}{ Nomenclature } \\
\hline $\boldsymbol{A}$ & Stretching stiffness matrix \\
\hline $\boldsymbol{C}_{R}, \boldsymbol{C}_{A}$ & Damping matrix \\
\hline$D$ & Bending stiffness matrix \\
\hline $\boldsymbol{D}_{\mathrm{a}}, \boldsymbol{D}_{\mathrm{s}}$ & Electric displacements \\
\hline$E_{\mathrm{b}}, E_{\mathrm{a}}, E_{\mathrm{s}}$ & Yong's modulus of the based layer, actuator layer and sensor layer \\
\hline $\boldsymbol{E}$ & Electric fields \\
\hline $\boldsymbol{F}$ & Point excitation \\
\hline$G_{F}$ & Constant gain \\
\hline $\boldsymbol{K}_{q q}, \boldsymbol{K}_{s p r}, \boldsymbol{K}_{q \psi}, \boldsymbol{K}_{\psi \psi}$ & $\begin{array}{l}\text { Generalized stiffness matrix, spring stiffness matrix, electrical mechanical coupled } \\
\text { stiffness, electrical stiffness matrix }\end{array}$ \\
\hline$L$ & Length of the shell \\
\hline $\boldsymbol{M}_{q q}$ & Generalized mass matrix \\
\hline $\boldsymbol{M}_{x}, \boldsymbol{M}_{\theta}, \boldsymbol{M}_{x \theta}$ & Moments of the in-plane stresses \\
\hline$N$ & Number of terms for circumferential wave \\
\hline$N_{x}, N_{\theta}, N_{x \theta}$ & Force of the in-plane stresses \\
\hline$N A$ & Number of supported point \\
\hline$N T$ & Number of terms for Chevbyshev polynomials \\
\hline $\boldsymbol{P}$ & Applied electrical charge for piezoelectric layers \\
\hline $\boldsymbol{Q}^{\mathrm{a}}, \boldsymbol{Q}^{\mathrm{s}}, \boldsymbol{Q}^{\mathrm{b}}$ & Plane stresses-strain matrix \\
\hline$Q_{\theta}, Q_{x}$ & Transverse shear force \\
\hline$Q_{N}$ & Nonlinear item \\
\hline$R$ & Radius of the shell \\
\hline$T$ & Kinetic energy \\
\hline$T_{m}^{*}(\xi)$ & Admissible displacement functions \\
\hline$U_{\varepsilon}, U_{\mathrm{spr}}$ & Strain energy, potential energy \\
\hline $\bar{U}, \bar{V}, \bar{W}, \bar{\Phi}_{x}, \bar{\Phi}_{\theta}$ & Mode vector satisfying the boundary condition \\
\hline $\begin{array}{l}a_{m n}, b_{m n}, c_{m n}, d_{m n} \\
e_{m n}, f_{m n}, g_{m n}\end{array}$ & Unknown corresponding coefficients \\
\hline $\boldsymbol{e}$ & Piezoelectric constants \\
\hline$f$ & Amplitude of excitation \\
\hline$h, h_{\mathrm{a}}, h_{\mathrm{s}}$ & Thickness of the shell of the based layer, actuator layer and sensor layer \\
\hline$k_{u}, k_{v}, k_{w}, k_{x}, k_{\theta}$ & Stiffness of axial, circumferential, radial, torsional spring \\
\hline$k_{\mathrm{c}}$ & Shear correction factor \\
\hline$n$ & Circumferential wave number \\
\hline$u, v, w, \phi_{x}, \phi_{\theta}$ & $\begin{array}{l}\text { Displacement in the } x, \theta, z \text { directions and rotations of the transverse normal respect to the } \\
x \text { and } \theta \text { axes }\end{array}$ \\
\hline$\overline{\boldsymbol{\Psi}}_{\mathrm{a}}, \overline{\boldsymbol{\Psi}}_{\mathrm{s}}$ & Distribution of electric potential along thickness coordinate \\
\hline $\boldsymbol{\Phi}$ & Mass normalized mode shape matrix \\
\hline$\varepsilon_{x}, \varepsilon_{\theta}, \gamma_{x \theta}, \gamma_{\theta z}, \gamma_{x z}$ & Strains of the shell \\
\hline$\zeta$ & Dielectric constants \\
\hline$\kappa_{x}, \kappa_{\theta}, \kappa_{x \theta}$ & Curvature of the shell \\
\hline$\mu_{\mathrm{b}}$ & Poisson's ratios of based layer \\
\hline$\psi$ & Values of electric potential of actuator and sensor layer \\
\hline$\xi$ & Non-dimensional axial coordinate \\
\hline$\left(\xi_{s}, \theta_{s}\right),\left(\xi_{s}^{\prime}, \theta_{s}^{\prime}\right)$ & Starting and ending coordinates of the $s$ th piezoelectric layer \\
\hline
\end{tabular}




\begin{tabular}{|ll|}
\hline$\rho_{\mathrm{b}}, \rho_{\mathrm{b}}, \rho_{\mathrm{b}}$ & Mass density of the based layer, actuator layer and sensor layer \\
$\sigma_{x}, \sigma_{\theta}, \tau_{x y}, \tau_{\theta y}, \tau_{x z}$ & Stresses of the shell \\
$\omega$ & Natural frequency of the shell \\
$\omega_{\mathrm{EX}}$ & Excitation frequency \\
\hline
\end{tabular}

\section{Theoretical formulations}

\subsection{Description of laminated cylindrical shells mode}

In Fig. 1, the schematic diagram of laminated cylindrical shells with discontinuous piezoelectric layer is presented, in which radius, length, thickness of the base layer, thickness of the piezoelectric sensor and actuator layers are defined as $R, L, h, h_{s}$ and $h_{a}$, respectively. Along the axial, circumferential and radial directions of the shell, an orthogonal coordinate system $(x, \theta, z)$ is established. In the $x, \theta, z$ directions, the displacements of a point on the middle surface are denoted as $u, v$ and $w$, in the rotations direction with respect to the $x$ and $\theta$ axes, the transverse normal are denoted as $\phi_{x}$ and $\phi_{\theta}$. The number of piezoelectric layers is defined as $N P$. The starting and ending coordinates of the $s$ th piezoelectric layer are $\left(\xi_{s}, \theta_{s}\right)$ and $\left(\xi_{s}^{\prime}, \theta_{s}^{\prime}\right)$, respectively. $N A$ is the number of restrained points. The arbitrary boundary conditions are represented by using artificial spring technique. $k_{u, \alpha}^{0}, k_{v, \alpha}^{0}, k_{w, \alpha}^{0}, k_{x, \alpha}^{0}$, and $k_{\theta, \alpha}^{0}$ are the stiffness of five groups of boundary springs at the $\alpha$ th point at $x=0$. Similarly, $k_{u, \alpha}^{1}, k_{v, \alpha}^{1}, k_{w, \alpha}^{1}, k_{x, \alpha}^{1}$ and $k_{\theta, \alpha}^{1}$ denote the stiffness of corresponding boundary springs.

(a)

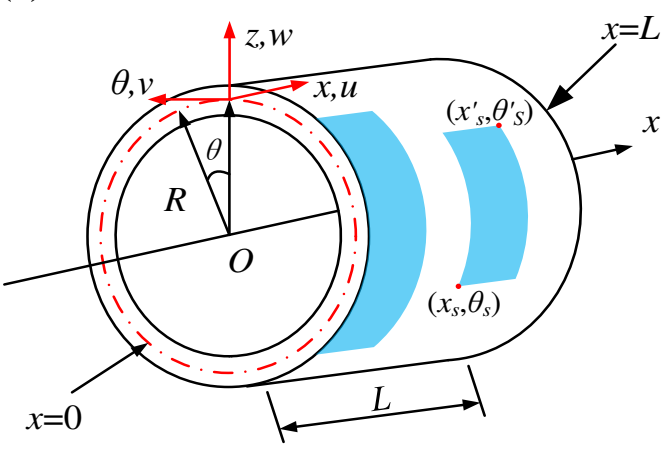

(b)

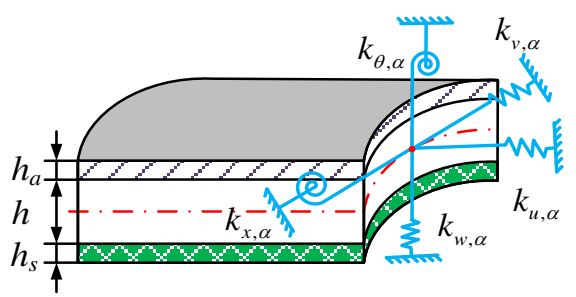

Fig. 1 Schematic diagram of a piezoelectric laminated composite cylindrical shell with elastic boundary conditions: (a) coordinate system and geometry of the shell; (b) partial cross-sectional view of the shell with elastic boundary condition 
4

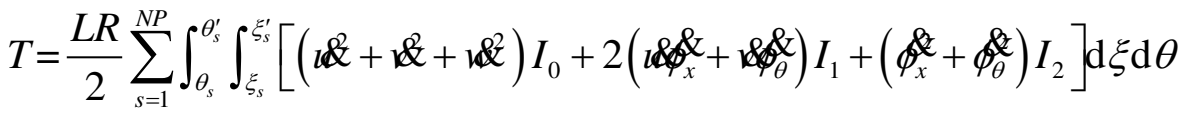

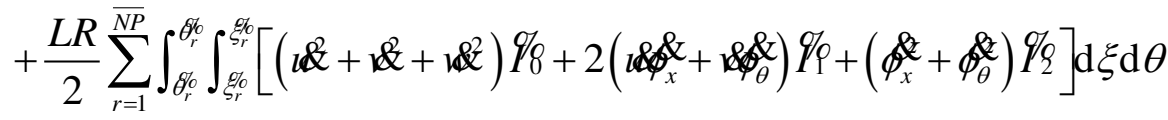

5 where,

6

$\left[I_{0}, I_{1}, I_{2}\right]=\int_{h / 2}^{h / 2+h_{\mathrm{a}}} \rho_{\mathrm{a}}\left[1, z, z^{2}\right] \mathrm{d} z+\int_{-h / 2}^{h / 2} \rho_{\mathrm{b}}\left[1, z, z^{2}\right] \mathrm{d} z+\int_{-h / 2-h_{\mathrm{s}}}^{-h / 2} \rho_{\mathrm{s}}\left[1, z, z^{2}\right] \mathrm{d} z$

$[\mathscr{P}, \mathscr{P}, \mathcal{P} /]=\int_{-h / 2}^{h / 2} \rho_{\mathrm{b}}\left[1, z, z^{2}\right] \mathrm{d} z$

7 where $\rho_{i}$ is the density of each layer of the shell. The subscript ( $i$ ) is used to represent the sensor layer

$8 \quad(i=\mathrm{s})$, actuator layer $(i=\mathrm{a})$, and base layer $(i=\mathrm{b})$.

$$
U=\frac{L R}{2} \sum_{s=1}^{N P} \int_{\theta_{s}}^{\theta_{s}^{\prime}} \int_{\xi_{s}}^{\xi_{s}^{\prime}}\left(N_{x}^{\mathrm{T}} \varepsilon_{x}+N_{\theta}^{\mathrm{T}} \varepsilon_{\theta}+N_{x \theta}^{\mathrm{T}} \gamma_{x \theta}+M_{x}^{\mathrm{T}} \kappa_{x}+M_{\theta}^{\mathrm{T}} \kappa_{\theta}+M_{x \theta}^{\mathrm{T}} \kappa_{x \theta}+Q_{\theta z}^{\mathrm{T}} \gamma_{\theta z}+Q_{x z}^{\mathrm{T}} \gamma_{x z}\right.
$$

9

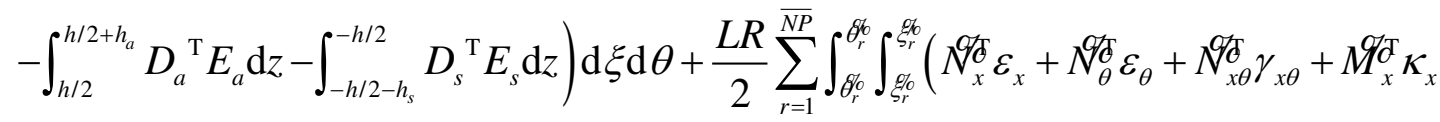

$$
\begin{aligned}
& \left.+M_{\theta}^{\partial} \kappa_{\theta}+M_{x \theta}^{\partial} \kappa_{x \theta}+Q_{\theta z}^{\Phi} \gamma_{\theta z}+Q_{x z}^{\Phi} \gamma_{x z}\right) \mathrm{d} \xi \mathrm{d} \theta
\end{aligned}
$$

10

11

12

where $\boldsymbol{D}_{i}$ and $\boldsymbol{E}_{i}$ are the electric displacements and electric fields of the piezoelectric layer[5],

respectively.

$$
\left\{\begin{array}{l}
D_{x}^{i} \\
D_{\theta}^{i} \\
D_{z}^{i}
\end{array}\right\}=\left[\begin{array}{lllll}
0 & 0 & 0 & 0 & e_{15 e}^{i} \\
0 & 0 & 0 & e_{24 e}^{i} & 0 \\
e_{31 e}^{i} & e_{32 e}^{i} & 0 & 0 & 0
\end{array}\right]\left\{\begin{array}{l}
\varepsilon_{x}^{i} \\
\varepsilon_{\theta}^{i} \\
\gamma_{x \theta}^{i} \\
\gamma_{\theta z}^{i} \\
\gamma_{x z}^{i}
\end{array}\right\}+\left[\begin{array}{ccc}
\zeta_{11 e}^{i} & 0 & 0 \\
0 & \zeta_{22 e}^{i} & 0 \\
0 & 0 & \zeta_{33 e}^{i}
\end{array}\right]\left\{\begin{array}{l}
E_{x}^{i} \\
E_{\theta}^{i} \\
E_{z}^{i}
\end{array}\right\}
$$

where $e_{i}$ and $\zeta_{i}$ are the piezoelectric constants and dielectric constants, respectively. 
$1 \quad E_{x}^{i}=-\frac{\partial \varphi^{i}(x, \theta, z, t)}{L \partial \xi}, E_{\theta}^{i}=-\frac{\partial \varphi^{i}(x, \theta, z, t)}{R \partial \theta}, E_{z}^{i}=-\frac{\partial \varphi^{i}(x, \theta, z, t)}{\partial z}$

2 where the piezoelectric layer potential due to elastic deformation is given as

3 $\varphi^{\mathrm{a}}(x, \theta, z, t)=\left[z_{\mathrm{a}}^{2}-\left(\frac{h_{\mathrm{a}}}{2}\right)^{2}\right] \psi_{\mathrm{a}}(x, \theta, t)$

$\varphi^{\mathrm{s}}(x, \theta, z, t)=\left[z_{\mathrm{s}}^{2}-\left(\frac{h_{\mathrm{s}}}{2}\right)^{2}\right] \psi_{\mathrm{s}}(x, \theta, t)$

4 where $\psi_{\mathrm{a}}(x, \theta, t)$ and $\psi_{\mathrm{s}}(x, \theta, t)$ are the distribution of the electric potential of the actuator layer along

5 the thickness coordinate, $z_{a}$ is the coordinate relative to the middle surface of the actuator layer,

$6 \quad z_{\mathrm{a}}=z-\left(h+h_{\mathrm{a}}\right) / 2 ; \psi_{\mathrm{s}}(x, \theta, t)$ is the distribution of the sensor layer, $z_{a}$ is the coordinate of the sensor

$7 \quad$ layer, $z_{\mathrm{s}}=z+\left(h+h_{\mathrm{s}}\right) / 2$.

$8 \quad$ In Eq.(3), the strain vector $\varepsilon^{\mathrm{T}}$ is given by[7]

$9 \quad \boldsymbol{\varepsilon}^{\mathrm{T}}=\left\{\begin{array}{llllllllll}\varepsilon_{x} & \varepsilon_{\theta} & \gamma_{x \theta} & \gamma_{\theta z} & \gamma_{x z} & \kappa_{x} & \kappa_{\theta} & \kappa_{x \theta} & \kappa_{x z} & \kappa_{\theta z}\end{array}\right\}$

The strains of a point of the shell are defined as[7]

$11 \quad\left(\begin{array}{l}\varepsilon_{x} \\ \varepsilon_{\theta} \\ \gamma_{x \theta} \\ \gamma_{\theta z} \\ \gamma_{x z}\end{array}\right)=\left(\begin{array}{l}\varepsilon_{x} \\ \varepsilon_{\theta} \\ \gamma_{x \theta} \\ \gamma_{\theta z} \\ \gamma_{x z}\end{array}\right)_{(0)}+z\left(\begin{array}{l}\kappa_{x} \\ \kappa_{\theta} \\ \kappa_{x \theta} \\ \kappa_{x z} \\ \kappa_{\theta z}\end{array}\right)$

where the subscript (0) is the middle surface. The strain and the curvature can be given as[7]

$$
\varepsilon_{x(0)}=\frac{\partial u_{0}}{L \partial \xi}+\frac{1}{2 L^{2}}\left(\frac{\partial w_{0}}{\partial \xi}\right)^{2}, \quad \varepsilon_{\theta(0)}=\frac{1}{R} \frac{\partial v_{0}}{\partial \theta}+\frac{w_{0}}{R}+\frac{1}{2 R^{2}}\left(\frac{\partial w_{0}}{\partial \theta}\right)^{2},
$$

13

$\gamma_{x \theta(0)}=\frac{1}{R} \frac{\partial u_{0}}{\partial \theta}+\frac{\partial v_{0}}{L \partial \xi}+\frac{1}{L R}\left(\frac{\partial w_{0}}{\partial \xi} \frac{\partial w_{0}}{\partial \theta}\right), \quad \gamma_{\theta z(0)}=\phi_{\theta}+\frac{1}{R} \frac{\partial w_{0}}{\partial \theta}-\frac{v_{0}}{R}, \quad \gamma_{x z(0)}=\phi_{x}+\frac{1}{L} \frac{\partial w_{0}}{\partial \xi}$

$\kappa_{x}=\frac{\partial \phi_{x}}{L \partial \xi}, \quad \kappa_{\theta}=\frac{1}{R} \frac{\partial \phi_{\theta}}{\partial \theta}, \quad \kappa_{x \theta}=\frac{1}{L} \frac{\partial \phi_{\theta}}{\partial \xi}+\frac{1}{R} \frac{\partial \phi_{x}}{\partial \theta}, \quad \kappa_{x z}=0, \quad \kappa_{\theta z}=0$

In the model, the position at which the piezoelectric layer is considered as a laminated shell, and the position at which the piezoelectric layer is not disposed is regarded as a single thin-wall cylindrical 
1 shell. In the location of the base layer, the matrix form of the force and moment resultant relations to

2 the strains in the middle surface and curvature changes are defined as[8]

$$
\begin{aligned}
& \left\{\begin{array}{l}
M_{x}^{o} \\
M_{\theta}^{o} \\
M_{x \theta}^{o} \\
M_{x}^{o} \\
M_{\theta}^{o} \\
M_{x \theta}^{o}
\end{array}\right\}=\left[\begin{array}{llllll}
A_{11}^{o} & A_{12}^{o} & 0 & B_{11}^{o} & B_{12}^{o} & 0 \\
A_{12}^{o} & A_{22}^{o} & 0 & B_{12}^{o} & B_{22}^{o} & 0 \\
0 & 0 & A_{66}^{o} & 0 & 0 & B_{66}^{o} \\
B_{11}^{o} & B_{12}^{o} & 0 & B_{11}^{o} & B_{12}^{o} & 0 \\
B_{12}^{o} & B_{22}^{o} & 0 & B_{12}^{o} & B_{22}^{o} & 0 \\
0 & 0 & B_{66}^{o} & 0 & 0 & B_{66}^{o}
\end{array}\right]\left\{\begin{array}{l}
\varepsilon_{x(0)} \\
\varepsilon_{\theta(0)} \\
\gamma_{x \theta(0)} \\
\kappa_{x} \\
\kappa_{\theta} \\
\kappa_{x \theta}
\end{array}\right\} \\
& \left\{\begin{array}{l}
\mathscr{Q}_{\theta}^{o} \\
\mathscr{Q}_{x}^{o}
\end{array}\right\}=k_{\mathrm{c}}\left[\begin{array}{cc}
\mathscr{A}_{44}^{o} & 0 \\
0 & \not_{55}^{o}
\end{array}\right]\left\{\begin{array}{l}
\gamma_{\theta z(0)} \\
\gamma_{x z(0)}
\end{array}\right\}
\end{aligned}
$$

$4 \quad$ where, $\AA_{i j}^{o}, B_{i j}^{o}$ and $B_{i j}^{o}$ are the stretching, coupling and bending stiffness matrixes, respectively. $k_{\mathrm{c}}$ is

5 shear correction factor, generally taken as 5/6.

$\AA_{i j}^{o}=\int_{-h / 2}^{h / 2} Q_{i j}^{\mathrm{b}} \mathrm{d} z$

$6 \quad B_{i j}^{o}=\int_{-h / 2}^{h / 2} Q_{i j}^{\mathrm{b}} z \mathrm{~d} z$

$$
B_{i j}^{O}=\int_{-h / 2}^{h / 2} Q_{i j}^{\mathrm{b}} z^{2} \mathrm{~d} z
$$

$7 \quad$ where the plane stresses-strain matrix $\boldsymbol{Q}_{\mathrm{b}}$ is as[7]

$8 \quad Q_{\mathrm{b}}=\left[\begin{array}{lllll}Q_{11}^{\mathrm{b}} & Q_{12}^{\mathrm{b}} & 0 & 0 & 0 \\ Q_{12}^{\mathrm{b}} & Q_{22}^{\mathrm{b}} & 0 & 0 & 0 \\ 0 & 0 & Q_{66}^{\mathrm{b}} & 0 & 0 \\ 0 & 0 & 0 & Q_{44}^{\mathrm{b}} & 0 \\ 0 & 0 & 0 & 0 & Q_{55}^{\mathrm{b}}\end{array}\right]$

$9 \quad$ where the elements of $Q_{i j}^{\mathrm{b}}$ are given as

$$
Q_{11}^{\mathrm{b}}=Q_{22}^{\mathrm{b}}=\frac{E_{\mathrm{b}}}{1-\mu_{\mathrm{b}}^{2}}, \quad Q_{12}^{\mathrm{b}}=Q_{21}^{\mathrm{b}}=\frac{\mu_{\mathrm{b}} E_{\mathrm{b}}}{1-\mu_{\mathrm{b}}^{2}}, \quad Q_{66}^{\mathrm{b}}=\frac{E_{\mathrm{b}}}{2\left(1+\mu_{\mathrm{b}}\right)}, \quad Q_{44}^{\mathrm{b}}=Q_{66}^{\mathrm{b}}, \quad Q_{55}^{\mathrm{b}}=Q_{66}^{\mathrm{b}}
$$

11 where $E_{\mathrm{b}}$ is Yong's moduli of the base layer. $\mu_{\mathrm{b}}$ is the Poisson's ratios.

In the location of base-piezoelectric layers, the matrix form of the force and moment resultant relations to the strains in the middle surface and curvature changes are defined as[8] 


$$
\begin{aligned}
& \left\{\begin{array}{l}
N_{x} \\
N_{\theta} \\
N_{x \theta} \\
M_{x} \\
M_{\theta} \\
M_{x \theta}
\end{array}\right\}=\left[\begin{array}{llllll}
A_{11} & A_{12} & 0 & B_{11} & B_{12} & 0 \\
A_{12} & A_{22} & 0 & B_{12} & B_{22} & 0 \\
0 & 0 & A_{66} & 0 & 0 & B_{66} \\
B_{11} & B_{12} & 0 & D_{11} & D_{12} & 0 \\
B_{12} & B_{22} & 0 & D_{12} & D_{22} & 0 \\
0 & 0 & B_{66} & 0 & 0 & D_{66}
\end{array}\right]\left\{\begin{array}{l}
\varepsilon_{x(0)} \\
\varepsilon_{\theta(0)} \\
\gamma_{x \theta(0)} \\
\kappa_{x} \\
\kappa_{\theta} \\
\kappa_{x \theta}
\end{array}\right\}+\left\{\begin{array}{l}
N_{x}^{\mathrm{E}} \\
N_{\theta}^{\mathrm{E}} \\
N_{x \theta}^{\mathrm{E}} \\
M_{x}^{\mathrm{E}} \\
M_{\theta}^{\mathrm{E}} \\
M_{x \theta}^{\mathrm{E}}
\end{array}\right\} \\
& \left\{\begin{array}{l}
Q_{\theta} \\
Q_{x}
\end{array}\right\}=k_{\mathrm{c}}\left[\begin{array}{ll}
A_{44} & 0 \\
0 & A_{55}
\end{array}\right]\left\{\begin{array}{l}
\gamma_{\theta z(0)} \\
\gamma_{x z(0)}
\end{array}\right\}+\left\{\begin{array}{l}
Q_{\theta}^{\mathrm{E}} \\
Q_{x}^{\mathrm{E}}
\end{array}\right\}
\end{aligned}
$$

2 where $N_{x}^{\mathrm{E}}, N_{\theta}^{\mathrm{E}}, N_{x \theta}^{\mathrm{E}}, M_{x}^{\mathrm{E}}, M_{\theta}^{\mathrm{E}}, M_{x \theta}^{\mathrm{E}}, Q_{\theta}^{\mathrm{E}}, Q_{x}^{\mathrm{E}}$ are the piezoelectric resultants of the piezoelectric layer.

$$
A_{i j}=\int_{h / 2}^{h / 2+h_{\mathrm{a}}} Q_{i j}^{\mathrm{a}} \mathrm{d} z+\int_{-h / 2}^{h / 2} Q_{i j}^{\mathrm{b}} \mathrm{d} z+\int_{-h / 2-h_{\mathrm{s}}}^{-h / 2} Q_{i j}^{\mathrm{s}} \mathrm{d} z
$$

$3 \quad B_{i j}=\int_{h / 2}^{h / 2+h_{i}} Q_{i j}^{\mathrm{a}} z \mathrm{~d} z+\int_{-h / 2}^{h / 2} Q_{i j}^{\mathrm{b}} z \mathrm{~d} z+\int_{-h / 2-h_{\mathrm{s}}}^{-h / 2} Q_{i j}^{\mathrm{s}} z \mathrm{~d} z$

$$
D_{i j}=\int_{h / 2}^{h / 2+h_{\mathrm{a}}} Q_{i j}^{\mathrm{a}} z^{2} \mathrm{~d} z+\int_{-h / 2}^{h / 2} Q_{i j}^{\mathrm{b}} z^{2} \mathrm{~d} z+\int_{-h / 2-h_{\mathrm{s}}}^{-h / 2} Q_{i j}^{\mathrm{s}} z^{2} \mathrm{~d} z
$$

$4 \quad$ where $\boldsymbol{Q}_{i}$ are the elastic constants.

5 The force and moment resultant of the piezoelectric layer are given as[5]

6

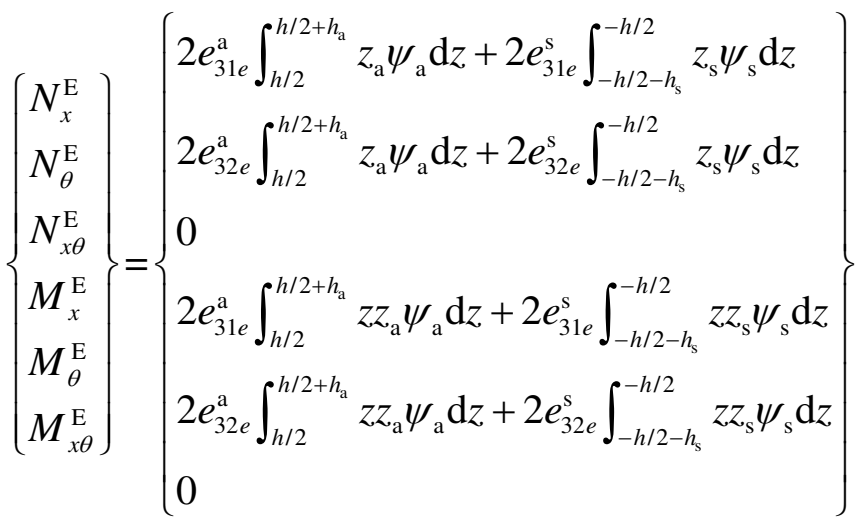

$$
\begin{aligned}
& \left\{\begin{array}{l}
Q_{\theta}^{\mathrm{E}} \\
Q_{x}^{\mathrm{E}}
\end{array}\right\}=\left\{\begin{array}{l}
e_{24 e}^{\mathrm{a}} \int_{h / 2}^{h / 2+h_{\mathrm{a}}} \frac{z_{\mathrm{a}}^{2}-\left(h_{\mathrm{a}} / 2\right)^{2}}{R+z} \frac{\partial \psi_{\mathrm{a}}}{\partial \theta} \mathrm{d} z+e_{24 e}^{s} \int_{-h / 2-h_{\mathrm{s}}}^{-h / 2}\left[\frac{z_{\mathrm{s}}^{2}-\left(h_{\mathrm{s}} / 2\right)^{2}}{R+z}\right] \mathrm{d} z \frac{\partial \psi_{\mathrm{s}}}{\partial \theta} \\
e_{15 e}^{\mathrm{a}} \int_{h / 2}^{h / 2+h_{\mathrm{a}}}\left[z_{a}^{2}-\left(h_{\mathrm{a}} / 2\right)^{2}\right] \frac{\partial \psi_{\mathrm{a}}}{\partial x} \mathrm{~d} z+e_{15 e}^{\mathrm{s}} \int_{-h / 2-h_{\mathrm{s}}}^{-h / 2}\left[z_{\mathrm{s}}{ }^{2}-\left(h_{\mathrm{s}} / 2\right)^{2}\right] \mathrm{d} z \frac{\partial \psi_{\mathrm{s}}}{\partial x}
\end{array}\right\}
\end{aligned}
$$

\subsection{The potential energy of the boundary springs}

To simulate arbitrary boundary conditions, we use a series of artificial spring in five directions.

The potential energy $U_{\mathrm{spr}}$ introduced by the elastic boundary springs can be calculated as follows[25] 
1

$$
\begin{aligned}
U_{\mathrm{spr}}= & \frac{1}{2} \sum_{\alpha=1}^{N A}\left\{k_{u, \alpha}^{0}\left[u\left(0, \theta_{\alpha}(t), t\right)\right]^{2}+k_{v, \alpha}^{0}\left[v\left(0, \theta_{\alpha}(t), t\right)\right]^{2}+k_{w, \alpha}^{0}\left[w\left(0, \theta_{\alpha}(t), t\right)\right]^{2}\right. \\
& \left.+k_{x, \alpha}^{0}\left[\phi_{x}\left(0, \theta_{\alpha}(t), t\right)\right]^{2}+k_{\theta, \alpha}^{0}\left[\phi_{\theta}\left(0, \theta_{\alpha}(t), t\right)\right]^{2}\right\} \\
& +\frac{1}{2} \sum_{\alpha=1}^{N A}\left\{k_{u, \alpha}^{1}\left[u\left(1, \theta_{\alpha}(t), t\right)\right]^{2}+k_{v, \alpha}^{1}\left[v\left(1, \theta_{\alpha}(t), t\right)\right]^{2}+k_{w, \alpha}^{1}\left[w\left(1, \theta_{\alpha}(t), t\right)\right]^{2}\right. \\
& \left.+k_{x, \alpha}^{1}\left[\phi_{x}\left(1, \theta_{\alpha}(t), t\right)\right]^{2}+k_{\theta, \alpha}^{1}\left[\phi_{\theta}\left(1, \theta_{\alpha}(t), t\right)\right]^{2}\right\}
\end{aligned}
$$

2 where $\left(x_{\alpha}, \theta_{\alpha}\right)$ is used to represent the coordinates of the $s$ th point.

\subsection{Admissible displacement functions}

In Qin's study[6], the Chebyshev polynomials show the highest computational efficiency, the higher convergence rate and the higher computational efficiency, compared with the Orthogonal polynomials and the modified Fourier series. Therefore, the admissible displacement functions in this paper use the Chebyshev polynomials. Then, the admissible displacement function is given by

$$
\left\{\begin{array}{l}
u(\xi, \theta, t)=\sum_{m=0}^{N T} \sum_{n=1}^{N} a_{m n} T_{m}^{*}(\xi) \cos n \theta e^{-j \omega t}=\overline{\boldsymbol{U}}^{\mathrm{T}} \boldsymbol{q}_{u} \\
v(\xi, \theta, t)=\sum_{m=0}^{N T} \sum_{n=1}^{N} b_{m n} T_{m}^{*}(\xi) \sin n \theta e^{-j \omega t}=\overline{\boldsymbol{V}}^{\mathrm{T}} \boldsymbol{q}_{\boldsymbol{v}} \\
w(\xi, \theta, t)=\sum_{m=0}^{N T} \sum_{n=1}^{N} c_{m n} T_{m}^{*}(\xi) \cos n \theta e^{-j \omega t}+\sum_{m=0}^{N T} c_{m} T_{m}^{*}(\xi) e^{-j \omega t}=\overline{\boldsymbol{W}}^{\mathrm{T}} \boldsymbol{q}_{w} \\
\phi_{x}(\xi, \theta, t)=\sum_{m=1}^{N T} \sum_{n=1}^{N} d_{m n} T_{m}^{*}(\xi) \cos n \theta e^{-j \omega t}=\overline{\boldsymbol{\Phi}}_{x} \boldsymbol{q}_{\phi_{x}} \\
\phi_{\theta}(\xi, \theta, t)=\sum_{m=1}^{N T} \sum_{n=1}^{N} e_{m n} T_{m}^{*}(\xi) \sin n \theta e^{-j \omega t}=\overline{\boldsymbol{\Phi}}_{\theta} \boldsymbol{q}_{\phi_{\theta}} \\
\psi_{\mathrm{s}}(\xi, \theta, t)=\sum_{m=1}^{N T} \sum_{n=1}^{N} f_{m n} T_{m}^{*}(\xi) \cos n \theta e^{-j \omega t}=\overline{\boldsymbol{\Psi}}_{\mathrm{s}} \boldsymbol{q}_{\psi_{\mathrm{s}}} \\
\psi_{\mathrm{a}}(\xi, \theta, t)=\sum_{m=1}^{N T} \sum_{n=1}^{N} g_{m n} T_{m}^{*}(\xi) \cos n \theta e^{-j \omega t}=\overline{\boldsymbol{\Psi}}_{\mathrm{a}} \boldsymbol{q}_{\psi_{\mathrm{a}}}
\end{array}\right.
$$

9 where $a_{m n}, b_{m n}, c_{m n}, d_{m n}, e_{m n}, f_{m n}, g_{m n}$ are the unknown corresponding coefficients. $T_{m}^{*}(\xi)$ are appropriate admissible displacement functions[19], $N T$ is the number of terms of the admissible displacement functions. $\omega$ is the natural frequency. $\boldsymbol{q}_{u}, \boldsymbol{q}_{v}, \boldsymbol{q}_{w}, \boldsymbol{q}_{\phi_{x}}, \boldsymbol{q}_{\phi_{\theta}}, \boldsymbol{q}_{\psi_{\mathrm{s}}}, \boldsymbol{q}_{\psi_{\mathrm{a}}}$ are the generalized 
1 the boundary condition.

6

7

$$
U_{\mathrm{spr}}=\frac{1}{2} \boldsymbol{q}_{u}^{\mathrm{T}} \boldsymbol{K}_{\mathrm{spr}}^{u u} \boldsymbol{q}_{u}+\frac{1}{2} \boldsymbol{q}_{v}^{\mathrm{T}} \boldsymbol{K}_{\mathrm{spr}}^{v v} \boldsymbol{q}_{v}+\frac{1}{2} \boldsymbol{q}_{w}^{\mathrm{T}} \boldsymbol{K}_{\mathrm{spr}}^{w w} \boldsymbol{q}_{w}+\frac{1}{2} \boldsymbol{q}_{\phi_{x}}^{\mathrm{T}} \boldsymbol{K}_{\mathrm{spr}}^{\phi_{x} \phi_{x}} \boldsymbol{q}_{\phi_{x}}+\frac{1}{2} \boldsymbol{q}_{\phi_{\theta}}^{\mathrm{T}} \boldsymbol{K}_{\mathrm{spr}}^{\phi_{\phi} \phi_{\theta}} \boldsymbol{q}_{\phi_{\theta}}
$$

8 The Lagrange equation is given by

9

$$
\begin{aligned}
& \left(\frac{\mathrm{d}}{\mathrm{d} t}\left(\frac{\partial T}{\partial \boldsymbol{q}_{u}^{\&}}\right)-\frac{\partial T}{\partial \boldsymbol{q}_{u}}+\frac{\partial\left(U_{\varepsilon}+U_{\mathrm{spr}}\right)}{\partial \boldsymbol{q}_{u}}+\frac{\partial D}{\partial \boldsymbol{q}_{u}^{\&}}=\boldsymbol{f}_{u}\right. \\
& \frac{\mathrm{d}}{\mathrm{d} t}\left(\frac{\partial T}{\partial \boldsymbol{q}_{v}}\right)-\frac{\partial T}{\partial \boldsymbol{q}_{v}}+\frac{\partial\left(U_{\varepsilon}+U_{\mathrm{spr}}\right)}{\partial \boldsymbol{q}_{v}}+\frac{\partial D}{\partial \boldsymbol{q}_{v}^{\mathrm{K}}}=\boldsymbol{f}_{v} \\
& \left\{\begin{array}{l}
\frac{\mathrm{d}}{\mathrm{d} t}\left(\frac{\partial T}{\partial \boldsymbol{q}_{w}^{\mathrm{k}}}\right)-\frac{\partial T}{\partial \boldsymbol{q}_{w}}+\frac{\partial\left(U_{\varepsilon}+U_{\mathrm{spr}}\right)}{\partial \boldsymbol{q}_{w}}+\frac{\partial D}{\partial \boldsymbol{q}_{w}^{\mathrm{k}}}=\boldsymbol{f}_{w} \\
\frac{\mathrm{d}}{\mathrm{d} t}\left(\frac{\partial T}{\partial \boldsymbol{q}_{\boldsymbol{\phi}_{x}}^{\mathrm{K}}}\right)-\frac{\partial T}{\partial \boldsymbol{q}_{\phi_{x}}}+\frac{\partial\left(U_{\varepsilon}+U_{\mathrm{spr}}\right)}{\partial \boldsymbol{q}_{\phi_{x}}}+\frac{\partial D}{\partial \boldsymbol{q}_{\boldsymbol{\phi}_{x}}}=\boldsymbol{f}_{\phi_{x}}
\end{array}\right. \\
& \frac{\mathrm{d}}{\mathrm{d} t}\left(\frac{\partial T}{\partial \boldsymbol{q}_{\phi_{\theta}}^{\mathrm{z}}}\right)-\frac{\partial T}{\partial \boldsymbol{q}_{\phi_{\theta}}}+\frac{\partial\left(U_{\varepsilon}+U_{\mathrm{spr}}\right)}{\partial \boldsymbol{q}_{\phi_{\theta}}}+\frac{\partial D}{\partial \boldsymbol{q}_{\phi_{\theta}}^{\mathrm{x}}}=\boldsymbol{f}_{\phi_{\theta}} \\
& \frac{\mathrm{d}}{\mathrm{d} t}\left(\frac{\partial T}{\partial \boldsymbol{\psi}_{a}^{\mathrm{\alpha}}}\right)-\frac{\partial T}{\partial \boldsymbol{\psi}_{a}}+\frac{\partial\left(U_{\varepsilon}+U_{\mathrm{spr}}\right)}{\partial \boldsymbol{\psi}_{a}}+\frac{\partial D}{\partial \boldsymbol{\psi}_{a}^{\mathrm{k}}}=\boldsymbol{p}_{a}
\end{aligned}
$$


$1 \quad\left\{\frac{\mathrm{d}}{\mathrm{d} t}\left(\frac{\partial T}{\partial \boldsymbol{\psi}_{s}}\right)-\frac{\partial T}{\partial \boldsymbol{\psi}_{s}}+\frac{\partial\left(U_{\varepsilon}+U_{\mathrm{spr}}\right)}{\partial \boldsymbol{\psi}_{s}}+\frac{\partial D}{\partial \boldsymbol{\psi}_{s}}=\boldsymbol{p}_{s}\right.$

2 For the piezoelectric laminated cylindrical shell, the differential equations of electro-elastic

3 coupling motion included the elastic displacements $\boldsymbol{q}$ and the electric potential vector $\boldsymbol{\psi}$ are given

4 by[26]

5

$\boldsymbol{M}_{q q} \boldsymbol{C}_{R} \boldsymbol{\phi}+\left(\boldsymbol{K}_{q q}+\boldsymbol{K}_{\mathrm{spr}}\right) \boldsymbol{q}+\boldsymbol{Q}_{N}+\boldsymbol{K}_{q \psi} \boldsymbol{\psi}=\boldsymbol{F}$

$\boldsymbol{K}_{q \psi}^{\mathrm{T}} \boldsymbol{q}-\boldsymbol{K}_{\psi \psi} \boldsymbol{\psi}=\boldsymbol{P}$

6 where $\boldsymbol{M}_{q q}, \boldsymbol{K}_{q q}$ and $\boldsymbol{K}_{\text {spr }}$ are the shell's mass matrix, stiffness matrix and spring stiffness matrix; $\boldsymbol{K}_{q \psi}$

7 is the coupled stiffness; $\boldsymbol{K}_{\psi \psi}$ is the electrical stiffness matrix; $\boldsymbol{Q}_{N}$ is the nonlinear item. These matrices

8 are given in Appendix A, B, C, D, respectively. $\boldsymbol{P}$ is applied electrical charge for the piezoelectric

9 layers, and $\boldsymbol{F}$ is the radial harmonic excitation on the position $(x, \theta)$, in which $\overline{\boldsymbol{W}}_{0}$ is the admissible

10 displacement functions in the shell's radial direction.

$\boldsymbol{q}=\left[\begin{array}{lllll}\boldsymbol{q}_{u} & \boldsymbol{q}_{v} & \boldsymbol{q}_{w} & \boldsymbol{q}_{\phi_{x}} & \boldsymbol{q}_{\phi_{\theta}}\end{array}\right]^{\mathrm{T}}, \boldsymbol{\psi}=\left[\begin{array}{ll}\boldsymbol{\psi}_{a} & \boldsymbol{\psi}_{s}\end{array}\right]^{\mathrm{T}}$

$\boldsymbol{P}=\left[\begin{array}{ll}\boldsymbol{p}_{a} & \boldsymbol{p}_{s}\end{array}\right], \boldsymbol{F}=\left[\begin{array}{lllll}\boldsymbol{f}_{u} & \boldsymbol{f}_{v} & \boldsymbol{f}_{w} & \boldsymbol{f}_{\phi_{x}} & \boldsymbol{f}_{\phi_{\theta}}\end{array}\right]^{\mathrm{T}}$

$11 \quad\left\{\begin{array}{l}\boldsymbol{f}_{u}(x, \theta, t)=\boldsymbol{O} \\ \boldsymbol{f}_{v}(x, \theta, t)=\boldsymbol{O} \\ \boldsymbol{f}_{w}(x, \theta, t)=\overline{\boldsymbol{W}}_{0} f \cos \omega_{\mathrm{EX}} t \\ \boldsymbol{f}_{\phi_{x}}(x, \theta, t)=\boldsymbol{O} \\ \boldsymbol{f}_{\phi_{\theta}}(x, \theta, t)=\boldsymbol{O}\end{array}\right.$

The differential equations of electro-elastic coupling motion can be written as following to facilitate the decoupling of the equation, this as

$$
\boldsymbol{M}_{q q} \boldsymbol{C}_{R} \boldsymbol{\alpha}+\left(\boldsymbol{K}_{q q}+\boldsymbol{K}_{s p r}\right) \boldsymbol{q}+\boldsymbol{Q}+\boldsymbol{K}_{q \psi}^{\mathrm{S}} \boldsymbol{\psi}_{s}+\boldsymbol{K}_{q \psi}^{\mathrm{A}} \boldsymbol{\psi}_{a}=\boldsymbol{F}
$$

$14 \quad \boldsymbol{K}_{q \psi}^{\mathrm{A} \mathrm{T}} \boldsymbol{q}-\boldsymbol{K}_{\psi \psi}^{\mathrm{A}} \boldsymbol{\psi}_{a}=\boldsymbol{p}_{a}$

$$
\boldsymbol{K}_{q \psi}^{\mathrm{S} \mathrm{T}} \boldsymbol{q}-\boldsymbol{K}_{\psi \psi}^{\mathrm{S}} \boldsymbol{\psi}_{s}=\boldsymbol{p}_{s}
$$




$$
\begin{aligned}
& \boldsymbol{M}_{q q} \boldsymbol{C}_{R} \boldsymbol{\alpha} \psi\left(\boldsymbol{K}_{q q}+\boldsymbol{K}_{s p r}+\boldsymbol{K}_{q \psi}^{\mathrm{S}} \boldsymbol{K}_{\psi \psi}^{\mathrm{S}-1} \boldsymbol{K}_{q \psi}^{\mathrm{S}}{ }^{\mathrm{T}}\right) \boldsymbol{q}+\boldsymbol{Q}_{N}=\boldsymbol{F}-\boldsymbol{K}_{q \psi}^{\mathrm{A}} \boldsymbol{\psi}_{a} \\
& \boldsymbol{\psi}_{s}=\boldsymbol{K}_{\psi \psi}^{\mathrm{S}-1} \boldsymbol{K}_{q \psi}^{\mathrm{S} \mathrm{T}} \boldsymbol{q}
\end{aligned}
$$

3 The negative velocity feedback control is used in this study as described in Fig. 2. The input

4 potential of the actuator layer is given as

$5 \quad \boldsymbol{\psi}_{a}=-G_{F} \psi_{S}^{\&}=-G_{F} \psi_{S}^{\&}=-G_{F} \boldsymbol{K}_{\psi \psi}^{\mathrm{S}}{ }^{-1}{ }^{\mathrm{S}}{ }_{q \psi}^{\mathrm{S}}{ }^{\mathrm{T}} \boldsymbol{\Phi}$

where $G_{F}$ is a constant gain. Then, the differential equation of decoupled motion write the next form

$$
\boldsymbol{M}_{q q}\left(\boldsymbol{C}_{A}+\boldsymbol{C}_{R}\right) \boldsymbol{d}+\left(\boldsymbol{K}_{q q}+\boldsymbol{K}_{s p r}+\boldsymbol{K}_{q \psi}^{S} \boldsymbol{K}_{\psi \psi}^{S-1} \boldsymbol{K}_{q \psi}^{S \mathrm{~T}}\right) \boldsymbol{q}+\boldsymbol{Q}_{N}=\boldsymbol{F}
$$

where $C_{R}$ is the Rayleigh damping matrix. $C_{A}$ is the damping matrix caused by the control potential.

$9 \quad C_{R}=\alpha \boldsymbol{M}_{q q}+\beta\left(\boldsymbol{K}_{q q}+\boldsymbol{K}_{s p r}\right)$

$C_{A}=-G_{\mathrm{F}} \boldsymbol{K}_{q \psi}^{A} \boldsymbol{K}_{\psi \psi}^{S-1} \boldsymbol{K}_{q \psi}^{S \mathrm{~T}}$

where $\alpha$ and $\beta$ are the Rayleigh damping parameters, which can be given as

12

$$
\begin{aligned}
& \alpha=2\left(\frac{\xi_{2}}{\omega_{2}^{2}}-\frac{\xi_{1}}{\omega_{1}^{2}}\right) /\left(\frac{1}{\omega_{2}^{2}}-\frac{1}{\omega_{1}^{2}}\right) \\
& \beta=2\left(\xi_{2} \omega_{2}-\xi_{1} \omega_{1}\right) /\left(\omega_{2}^{2}-\omega_{1}^{2}\right)
\end{aligned}
$$

where $\omega_{1}, \omega_{2}$ are the first and second order natural frequencies of the laminated cylindrical shells with discontinuous piezoelectric layer, $\xi_{1}$, and $\xi_{2}$ are the damp coefficient. The differential equation is solved by IHBM in this paper.

The vibration differential equations are solved to obtain the nonlinear response by using the Incremental Harmonic Balance Method. The specific process of IHBM is shown in Appendix E. 


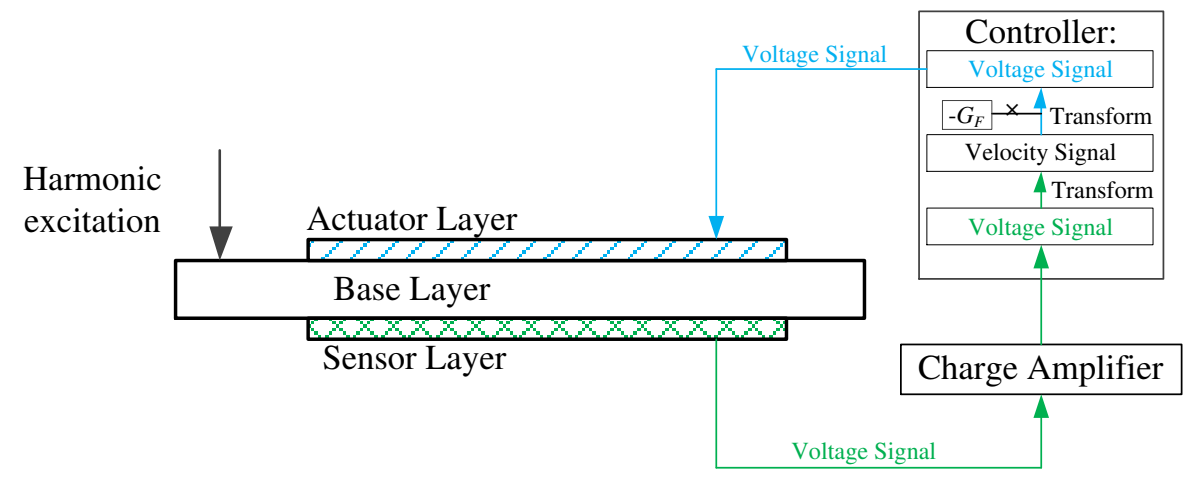

Fig. 2 The schematic diagram of feedback control

\section{Dynamic model verification}

\subsection{Linear model verification}

In the previous paper[8], we have studied the time-domain response of the discontinuous piezoelectric laminated cylindrical shells subject to pulse excitation, harmonic excitation, and optimized the position of the piezoelectric layer based on the system's retained energy. In the above study, the comparison with the finite element software ANSYS is sufficient to verify the accuracy of the linear part of the model. Therefore, we believed that the linear part of the model present in this paper is right, and the verification is no longer performed here.

\subsection{Nonlinear model verification}

In order to verify the nonlinear part of the model established in this paper, We compare the results obtained in this paper with those in literature $[9,10]$. The geometric parameters of the model in the verification process are: $L=0.09587 \mathrm{~m}, R=0.0678 \mathrm{~m}, H=0.678 \mathrm{~mm}$, and the damping coefficient $\xi=$ 0.001 has been used for $n=6$. The simply supported-simply supported boundary condition are employed, where $k_{u, \alpha}^{i}=k_{x, \alpha}^{i}=k_{\theta, \alpha}^{i}=0, k_{v, \alpha}^{i}=k_{w, \alpha}^{i}=10^{12}(i=0,1)$. In the response curve in Fig. 3, $\omega$ is the excitation frequency, $\omega_{(1,6)}$ is the fundamental frequency $(m=1, n=6), A$ is the amplitude of the mode, and $H$ denotes the thickness of the shell. 


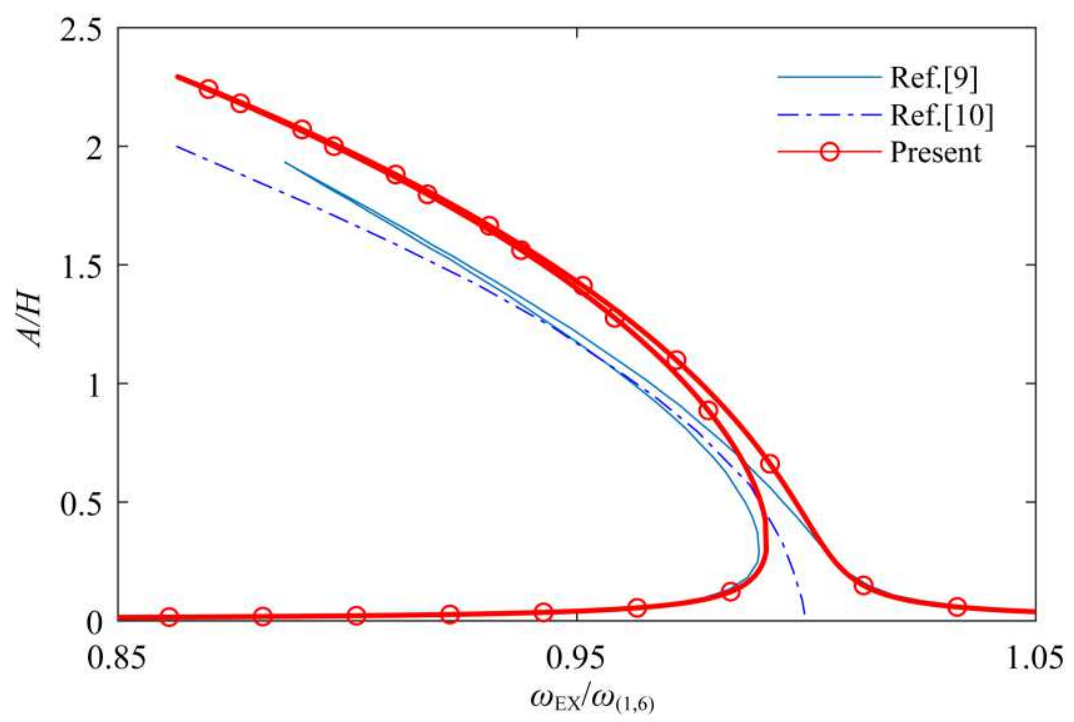

Fig. 3 Comparison of amplitude-frequency curves with the references

It can be seen from the Fig. 3 that the amplitude-frequency curves obtained in this paper have a similar variation trend with those in the references. After the verification of both linear and nonlinear parts, it can be considered that the method we used is correct.

\section{Results and Discussions}

In this section, the IHBM is used to obtain the nonlinear frequency-amplitude response curves of the piezoelectric laminated cylindrical shell. The structural parameters are selected as follows: $h=0.002 \mathrm{~m}, h_{a}=0.001 \mathrm{~m}, h_{s}=0.001 \mathrm{~m}, L=0.1 \mathrm{~m}, R=0.1 \mathrm{~m}, N T=5, f=2 \mathrm{e} 3 \mathrm{~N}, N A=16, k_{u, \alpha}^{1}=k_{v, \alpha}^{1}=k_{w, \alpha}^{1}=k_{\theta, \alpha}^{1}=$ $k_{x, \alpha}^{1}=1 \mathrm{e} 12 \mathrm{~N} / \mathrm{m}(\mathrm{N} / \mathrm{rad}), \quad k_{u, \alpha}^{0}=k_{v, \alpha}^{0}=k_{w, \alpha}^{0}=k_{\theta, \alpha}^{0}=k_{x, \alpha}^{0}=0 \mathrm{~N} / \mathrm{m}(\mathrm{N} / \mathrm{rad})$, the exciting position $(x, \theta)$ is $(0.9,3)$, the response position is $(0.9,3.2)$. In order to facilitate the comparison of different results, the horizontal and vertical coordinates of the amplitude-frequency response curve are dimensionless, that is, the $x$-axis is $\omega / \omega_{1,4}$ (In this paper, the established model reaches the minimum frequency when $n=4$, therefore, the first-order frequency $n=4$ is selected as the research object), the $y$-axis is $A / h$. The material parameters of the base layer and the piezoelectric layer are described in Table 1.

Table 1. Material properties of base layers and piezoelectric layers

\begin{tabular}{|c|c|c|c|c|c|c|c|}
\hline & & (GPa) & & $\rho_{\mathrm{b}}\left(\mathrm{kg} \cdot \mathrm{m}^{-3}\right)$ & & $\mu$ & \\
\hline \multirow[t]{2}{*}{ Base layer } & & 200 & & 7850 & & 0.26 & \\
\hline & $Q_{11}(\mathrm{GPa})$ & $Q_{12}(\mathrm{GPa})$ & $Q_{22}(\mathrm{GPa})$ & $Q_{44}(\mathrm{GPa})$ & $Q_{55}(\mathrm{GPa})$ & $Q_{66}(\mathrm{GPa})$ & $\rho_{\mathrm{e}}\left(\mathrm{kg} \cdot \mathrm{m}^{-3}\right)$ \\
\hline
\end{tabular}




\begin{tabular}{cccccccc} 
PVDF & 238.24 & 4. & 23.6 & 2.15 & 4.4 & 6.43 & 1800 \\
& $e_{31}\left(\mathrm{C} \cdot \mathrm{m}^{-2}\right)$ & $e_{32}\left(\mathrm{C} \cdot \mathrm{m}^{-2}\right)$ & $e_{24}\left(\mathrm{C} \cdot \mathrm{m}^{-2}\right)$ & $e_{15}\left(\mathrm{C} \cdot \mathrm{m}^{-2}\right)$ & $\zeta_{11}\left(10^{-11} \mathrm{~F} \cdot \mathrm{m}^{-1}\right)$ & $\zeta_{22}\left(10^{-11} \mathrm{~F} \cdot \mathrm{m}^{-1}\right)$ & $\zeta_{33}\left(10^{-11} \mathrm{~F} \cdot \mathrm{m}^{-1}\right)$ \\
\cline { 2 - 8 } PVDF & -0.13 & -0.14 & -0.01 & -0.01 & 0.885 & 0.885 & 10.6 \\
\hline
\end{tabular}

\subsection{Influence of the constant gain for frequency-amplitude response}

The effect of constant gain $G_{F}$ on the nonlinear amplitude-frequency response curve of a piezoelectric laminated cylindrical shell is discussed in this section. In Fig. 4, the range of the piezoelectric layer is: (a) the size and position of the randomly selected piezoelectric layer as $\xi \in[0.4,0.6], \theta \in[0,2 \pi]$; (b) the piezoelectric layer is completely covered on the surface of the shell, $\xi \in[0,1], \theta \in[0,2 \pi]$. The constant gain $G_{F}$ is set as $0,0.1,0.2,0.4$, respectively. According to Fig. 4, when the piezoelectric layer is completely covered on the surface of the shell, the nonlinear amplitude-frequency response curve has not obvious nonlinear characteristics, and approaches the linear amplitude-frequency response. As the constant gain $G_{F}$ increases, the response amplitude gradually decreases, and the nonlinear characteristics weaken, which proves that the piezoelectric layer has a large influence on the vibration response of the cylindrical shell.

When the position and size of the piezoelectric layer are random, as $\xi \in[0.4,0.6], \theta \in[0,2 \pi]$, the response curve has obvious soft nonlinear characteristics, and as the constant gain increases, the soft nonlinear characteristic weakens, and the amplitude decreases. After that, the time domain response when the excitation frequency is equal to the frequency at $\mathbf{A}$ or $\mathbf{B}$ (the peak frequency of nonlinear response when $G_{F}=0.4$ in Fig.4) is calculated in Fig. 5. It can be seen from Fig. 5 that the response tends to be stable with time, and the nonlinear response curve is no longer a symmetrical curve. 
4 Eq.(30) increases, the damping matrix $\boldsymbol{C}$ in Eq.(28) increases. However, the mass matrix and 5 stiffness matrix keep unchanged, therefore, the response amplitude decrease,

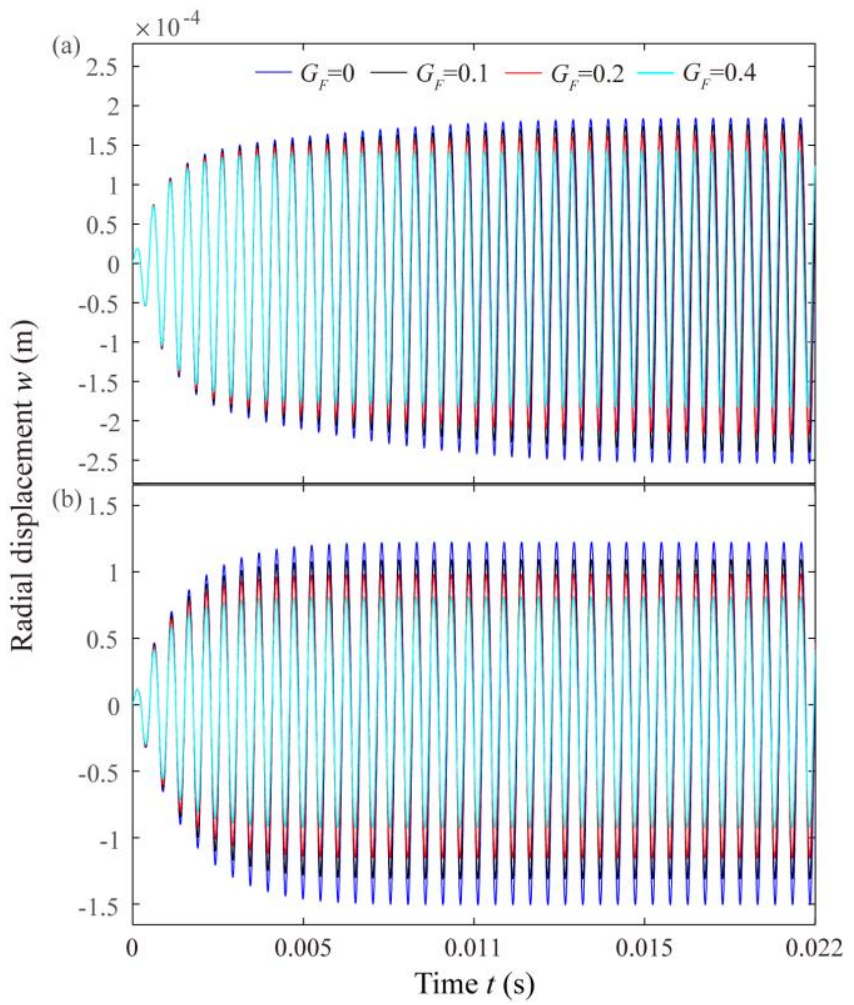

Fig. 4 The influence of the constant gain for frequency-amplitude response: (a) $\xi \in[0.4,0.6]$; (b) $\xi \in[0,1]$

The main reason for the phenomenon shown in Fig. 4 and Fig. 5 is that as the gain constant in Eq.(30) increases, the damping matrix $C$ in Eq.(28) increases. However, the mass matrix and

Fig. 5 The influence of the constant gain for time-domain response: (a) $\xi \in[0.4,0.6], \mathbf{A}: \omega_{\mathrm{EX}}=0.91 \omega_{1,4} ;$ (b) 8

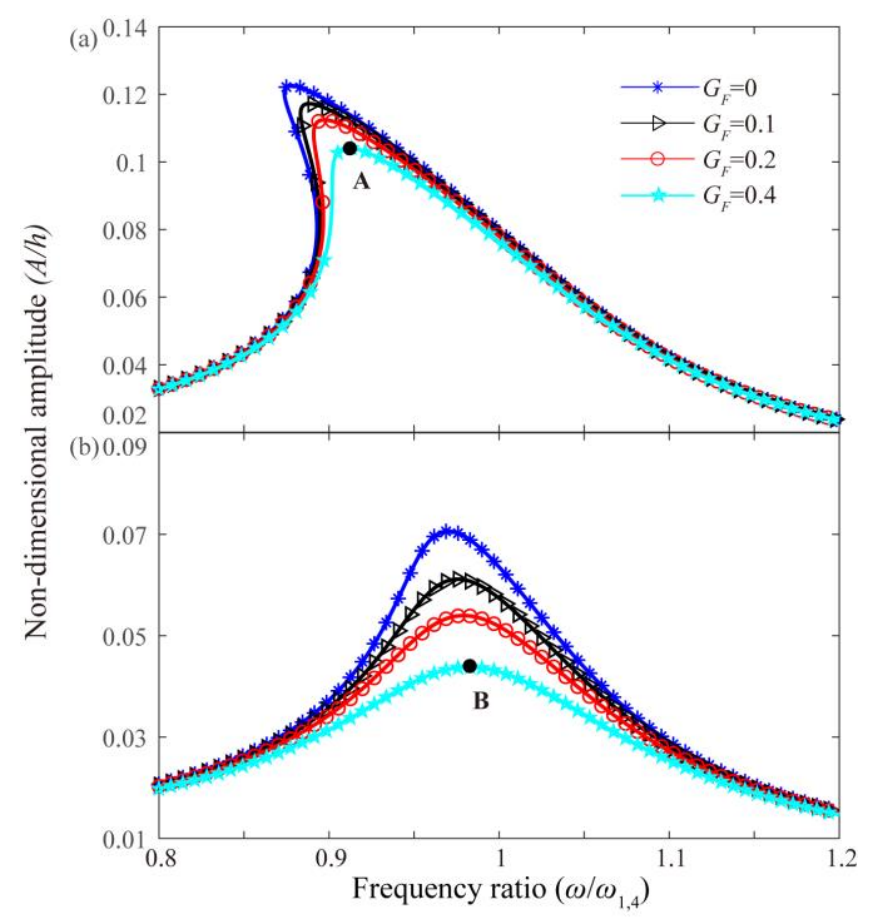

$$
\xi \in[0,1], \quad \mathbf{B}: \omega_{\mathrm{EX}}=0.98 \omega_{1,4}
$$




\subsection{Influence of the size of the piezoelectric layer for frequency-amplitude response}

According to the phenomenon in Sec. 4.1, we believe that it is necessary to study the influence of the size and position of the piezoelectric layer for the frequency-amplitude response. Then, the

effects of the size and position of the piezoelectric layer are analyzed. This section discusses the

effect of the size of the piezoelectric layer on the nonlinear amplitude-frequency response curve of the piezoelectric laminated cylindrical shell. In Fig. 6, the circumferential range of the piezoelectric layer is set as $\theta \in[0,2 \pi]$, the axial range is respectively set as $\xi \in[0,0.1], \xi \in[0,0.3], \xi \in[0,0.5]$,

$\xi \in[0,0.8], \xi \in[0,1]$, and the constant gain $G_{F}=0$ remains unchanged. It can be seen that the nonlinear amplitude-frequency response has obvious soft nonlinear characteristics, as the piezoelectric layer increases, the nonlinear characteristic of the response curve gradually decreases, and the response amplitude is reduced.

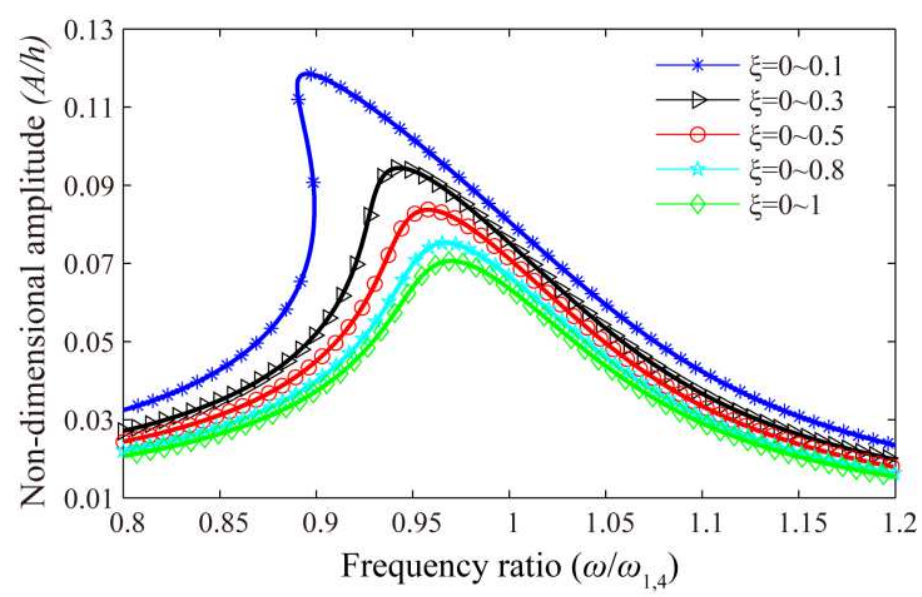

Fig. 6 The influence of the size of the piezoelectric layer for frequency-amplitude response 


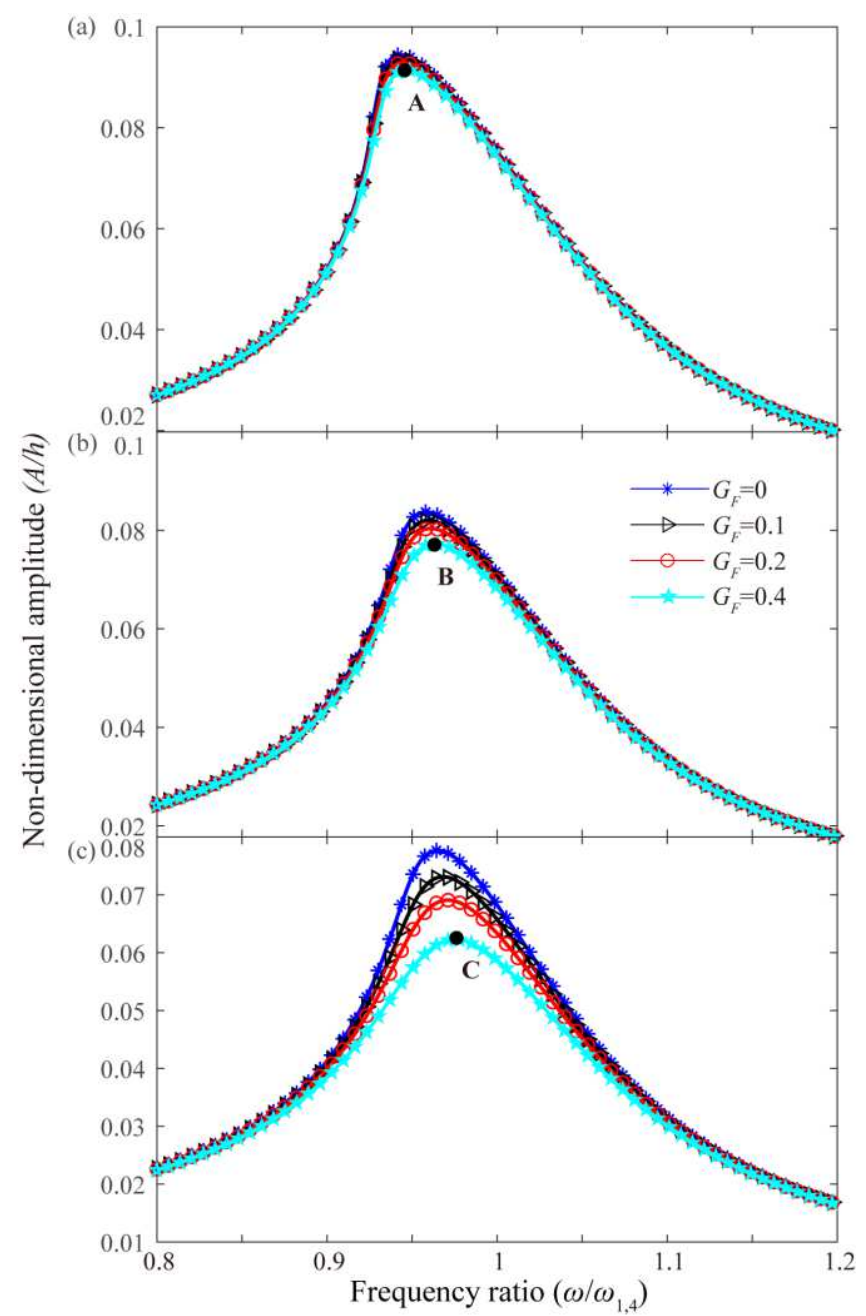

Fig. 7 The influences of the size of the piezoelectric layer and constant gain for frequency-amplitude response: (a)

$$
\xi \in[0,0.3] ; \text { (b) } \xi \in[0,0.5] ; \text { (c) } \xi \in[0,0.7]
$$

In Fig. 7, the effect of the piezoelectric layer's size on the nonlinear amplitude-frequency

response curve of a piezoelectric laminated cylindrical shell is analyzed for different constant gain, in which, $\xi \in[0,0.3], \xi \in[0,0.5], \xi \in[0,0.7]$. In each figure, the size of the piezoelectric layer is kept constant, and only the size of $G_{F}$ is changed. In Fig. $7(\mathrm{a}-\mathrm{c})$, when $\xi \in[0,0.3]$, the response curve changes less with the increase of $G_{F}$; when $\xi \in[0,0.7]$, the response curve obviously changes with different $G_{F}$. Then, the time domain response of different piezoelectric layers with frequencies at Point A, B and C (Fig. 7) are analyzed. The response curve tends to be stable with time, and the amplitude of the response curve decreases with the increase of constant gain. According to Figs. $6 \sim 8$, it can be seen that as the size of the piezoelectric layer increases, the amplitude of the 
nonlinear frequency-amplitude response curve decreases, and the nonlinear characteristic decreases; at the same time, the influence of the constant gain on the vibration response is weakened.

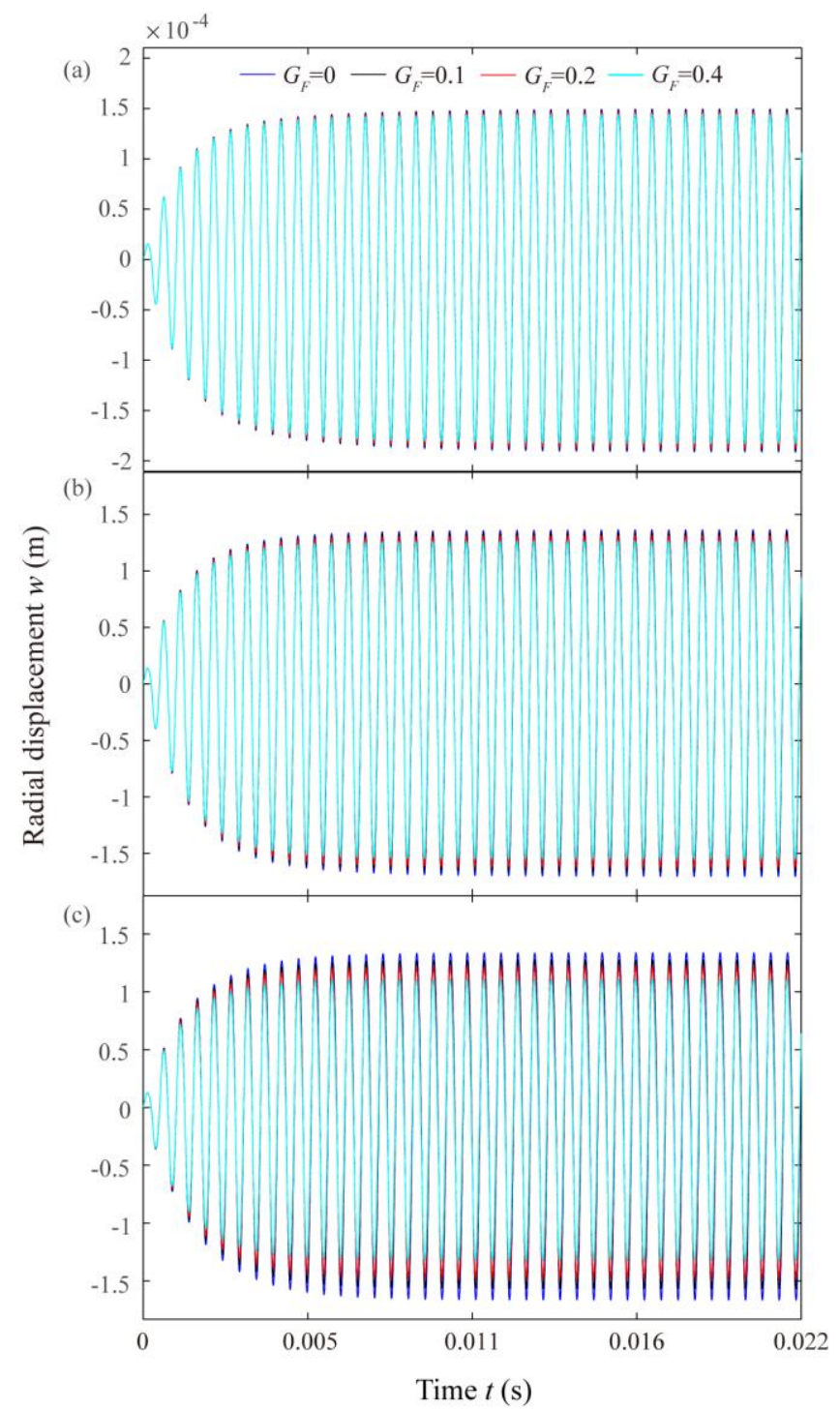

\subsection{Influence of the position of the piezoelectric layer for frequency-amplitude response}

This section discusses the effect of the position of the piezoelectric layer on the nonlinear

frequency-amplitude response curve of the piezoelectric laminated cylindrical shell with the clamped-free boundary condition. The size of the piezoelectric layer remains unchanged, $\xi \in[0,0.1], \theta \in[0,2 \pi]$. In Fig. 9, the position of the piezoelectric layer is respectively calculated as: $\xi \in[0,0.1] ; \quad \xi \in[0.5,0.6] ; \xi \in[0.5,0.6] ; \xi \in[0.7,0.8]$, and $G_{F}$ keeps unchanged. The nonlinear 
frequency-amplitude response of the piezoelectric laminated cylindrical shell has obvious soft nonlinear characteristics. When the position of the piezoelectric layer is at the clamped end, the nonlinear characteristic has a weaker amplitude; near the free end, the response's nonlinear characteristic is enhanced, and the amplitude is increased.

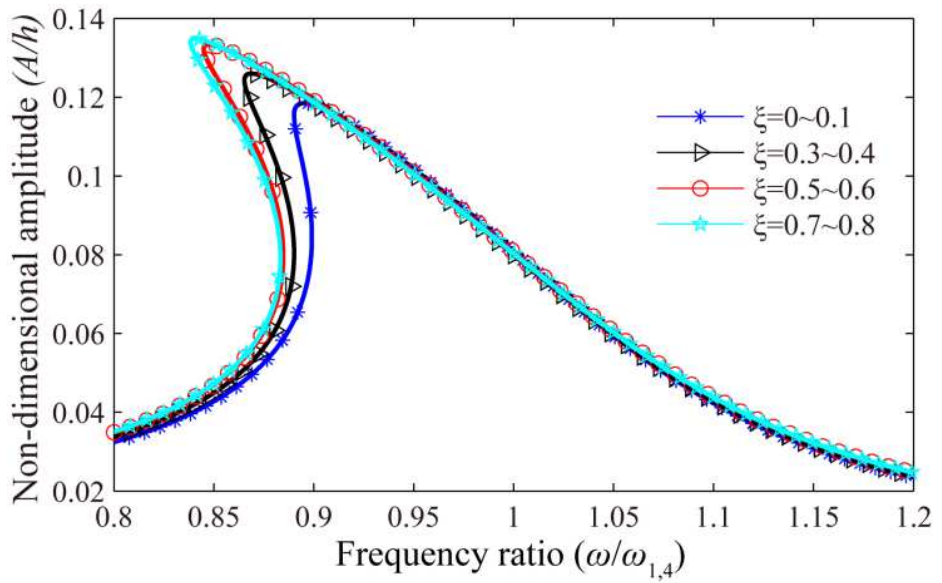

Fig. 9 The influence of the position of the piezoelectric layer for frequency-amplitude response

In Fig. 10, the influence of the positions of the piezoelectric layer on the nonlinear frequencyamplitude response curve of the piezoelectric laminated cylindrical shell at some different constant gain is analyzed. In each of the figures, the size and position of the piezoelectric layer are kept unchanged, only $G_{F}$ change. In Fig. $10(\mathrm{a} \sim \mathrm{c})$, when $\xi \in[0,0.1]$, as $G_{F}$ increases, the response curve changes less. When $\xi \in[0.7,0.8]$, with the rise of $G_{F}$, the amplitude and nonlinear characteristics of the response curve are obviously weakened. The time-domain response at different piezoelectric layer locations calculated in Fig. 11, the response curve has significant nonlinearity when the response is stabilized. It can be seen from Fig. 9 11 that as the position of the piezoelectric layer approaches the free end from the clamped end, the response amplitude increases, the nonlinear characteristic increases, and the effect of constant gain for the piezoelectric laminated cylindrical shell is enhanced. 


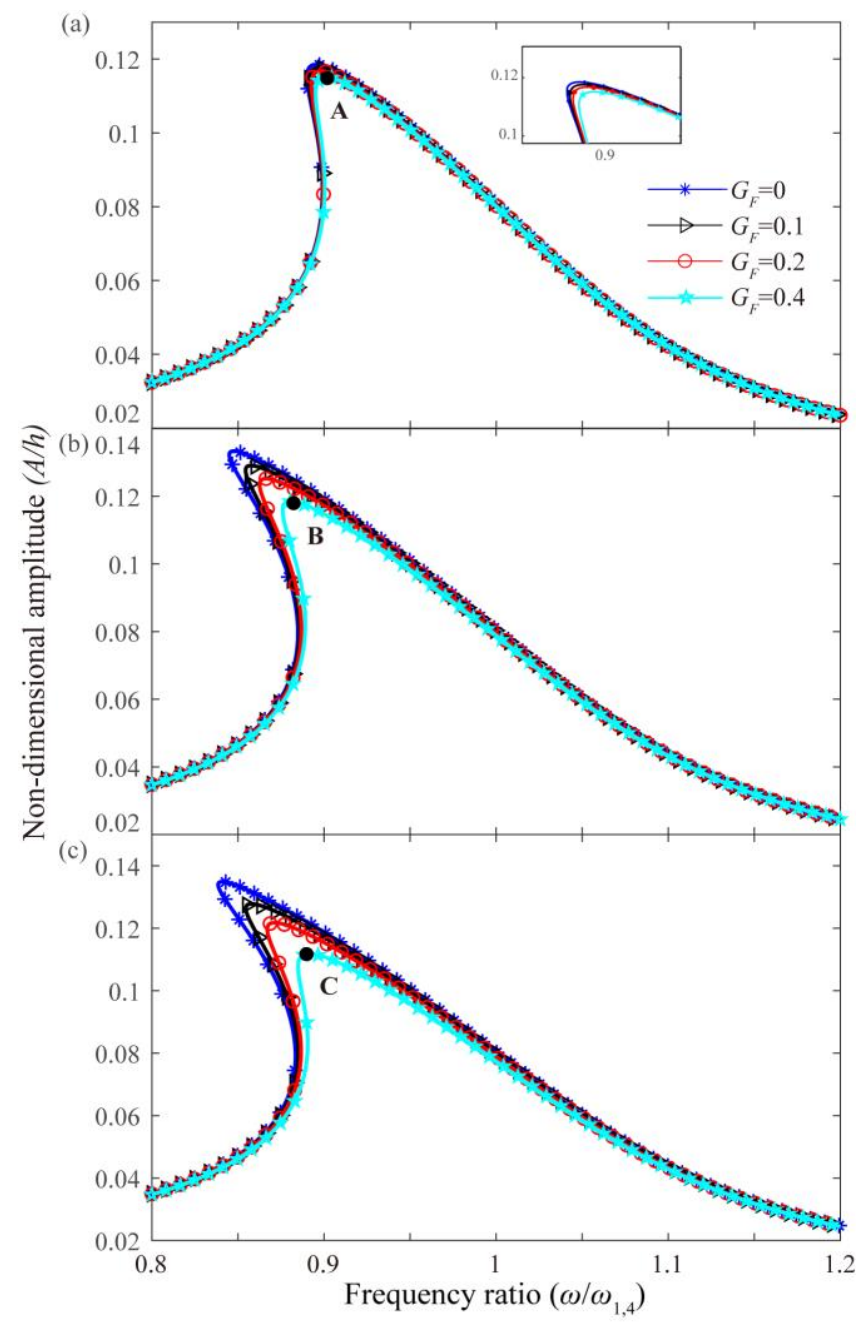

Fig. 10 The influences of the position of the piezoelectric layer and constant gain for frequency-amplitude response: (a) $\xi \in[0,0.1]$; (b) $\xi \in[0.5,0.6]$; (c) $\xi \in[0.7,0.8]$. 


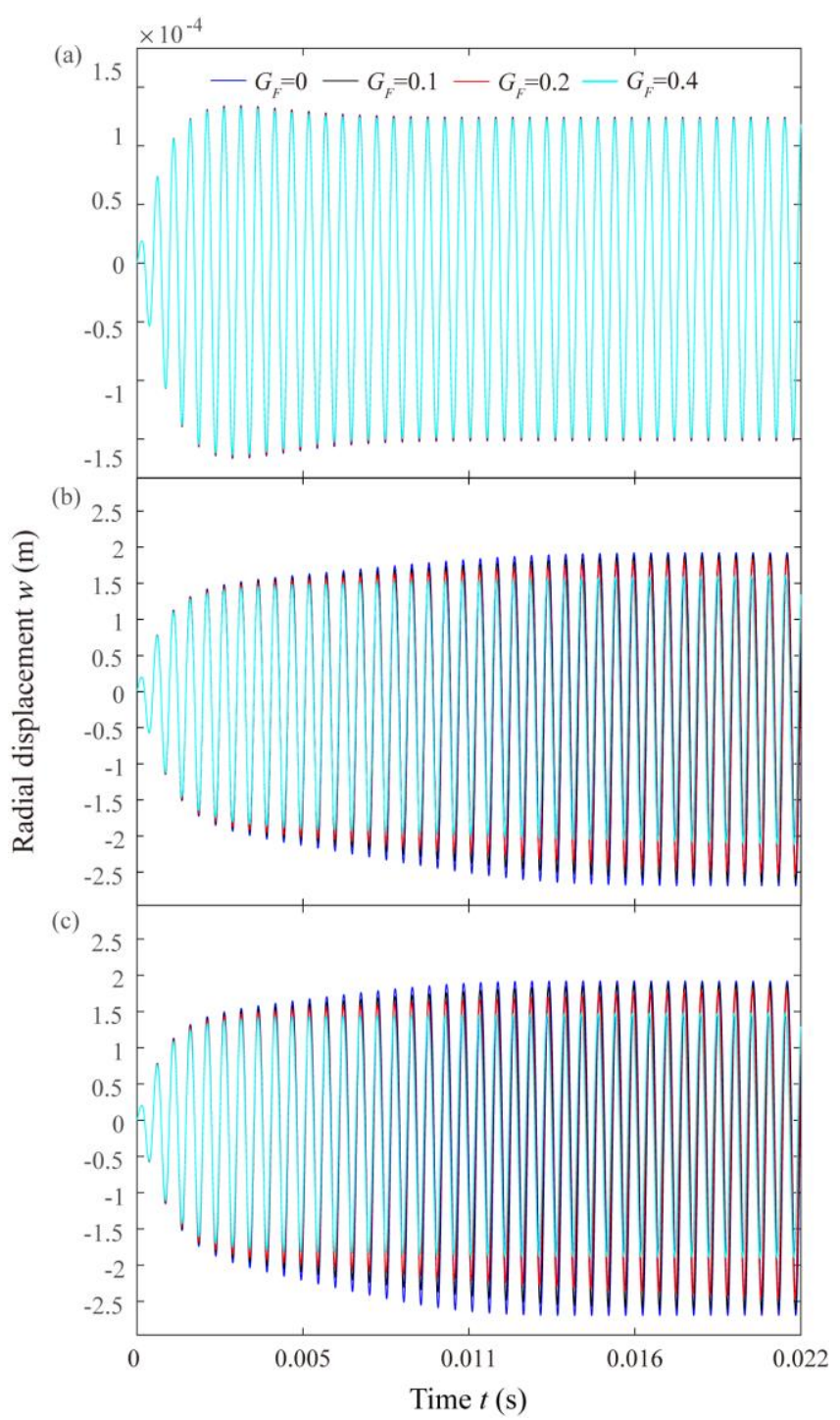

Fig. 11 The influences of the position of the piezoelectric layer and constant gain for time-domain response: (a)

$$
\xi \in[0,0.1], \mathbf{A}: \omega_{\mathrm{EX}}=0.91 \omega_{1,4} ; \text { (b) } \xi \in[0.5,0.6], \mathbf{B}: \omega_{\mathrm{EX}}=0.88 \omega_{1,4} ; \text { (c) } \xi \in[0.7,0.8], \mathbf{C}: \omega_{\mathrm{EX}}=0.89 \omega_{1,4}
$$

\section{Conclusions}

In this paper, we innovatively present a discontinuous piezoelectric laminated cylindrical shell model with point supported elastic boundary condition and study the effect of the discontinuous piezoelectric layer on geometric nonlinear vibration of the shell under radial harmonic excitation.

The following conclusions are drawn:

(1) With the increase of the constant gain $G_{F}$, the amplitude of the nonlinear frequency-amplitude response and time domain response decrease, at the same time, the nonlinear phenomenon weakens.

(2) As the size of the piezoelectric layer more prominent, the nonlinear phenomenon and the 
1 amplitude of the nonlinear response decrease.

2 (3) The effect of the piezoelectric layer on response is enhanced by constant gain $G_{F}$ when the 3 size increase.

4 (4) For the clamped-free piezoelectric laminated shells, the amplitude of amplitude-frequency

5 response increases slightly as the position of the piezoelectric layer is closer to the free end, and the

6 nonlinear characteristics are enhanced.

7 (5) The position of the piezoelectric layer is more obviously affected by the constant gain at the 8 free end.

\section{Acknowledgments} and the Fundamental Research Funds for the Central Universities (Nos. N180313008, N18241000706, and N170308028).

\section{Appendix A. Expressions for the mass matrix $M$}

$16 \quad \boldsymbol{M}_{q q}=\left[\begin{array}{lllll}\boldsymbol{M}^{u u} & 0 & 0 & \frac{1}{2} \boldsymbol{M}^{u \phi_{x}} & 0 \\ 0 & \boldsymbol{M}^{v v} & 0 & 0 & \frac{1}{2} \boldsymbol{M}^{v \phi_{\theta}} \\ 0 & 0 & \boldsymbol{M}^{w w} & 0 & 0 \\ \frac{1}{2} \boldsymbol{M}^{u \phi_{x} \mathrm{~T}} & 0 & 0 & \boldsymbol{M}^{\phi_{x} \phi_{x}} & 0 \\ 0 & \frac{1}{2} \boldsymbol{M}^{v \phi_{\theta} \mathrm{T}} & 0 & 0 & \boldsymbol{M}^{\phi_{\theta} \phi_{\theta}}\end{array}\right]$

where 


$$
\begin{aligned}
& \boldsymbol{M}^{u u}=L R \sum_{s=1}^{N P} \int_{\theta_{s}}^{\theta_{s}^{\prime}} \int_{\xi_{s}}^{\xi_{s}^{\prime}} \overline{\boldsymbol{U}} \overline{\boldsymbol{U}}^{\mathrm{T}} \mathrm{d} \xi \mathrm{d} \theta \cdot I_{0}+L R \sum_{r=1}^{\overline{N P}} \int_{\theta_{r}}^{\theta_{r}} \int_{\xi_{r}}^{\xi_{r}} \overline{\boldsymbol{U}} \overline{\boldsymbol{U}}^{\mathrm{T}} \mathrm{d} \xi \mathrm{d} \theta \cdot \mathscr{Y}_{8} \\
& \boldsymbol{M}^{u u}=L R \sum_{s=1}^{N P} \int_{\theta_{s}}^{\theta_{s}^{\prime}} \int_{\xi_{s}}^{\xi_{s}^{\prime}} \overline{\boldsymbol{U}} \overline{\boldsymbol{U}}^{\mathrm{T}} \mathrm{d} \xi \mathrm{d} \theta \cdot I_{0}+L R \sum_{r=1}^{\overline{N P}} \int_{\theta_{r}^{\prime}}^{\theta_{r}} \int_{\xi_{r}}^{\xi_{r}} \overline{\boldsymbol{U}} \overline{\boldsymbol{U}}^{\mathrm{T}} \mathrm{d} \xi \mathrm{d} \theta \cdot \mathscr{P} \\
& \boldsymbol{M}^{u \phi_{x}}=2 L R \sum_{s=1}^{N P} \int_{\theta_{s}}^{\theta_{s}^{\prime}} \int_{\xi_{s}}^{\xi_{s}^{\prime}} \overline{\boldsymbol{U}} \overline{\boldsymbol{\Phi}}_{x}^{\mathrm{T}} \mathrm{d} \xi \mathrm{d} \theta \cdot I_{1}+2 L R \sum_{r=1}^{\overline{N P}} \int_{\theta_{r}}^{\theta_{r}} \int_{\xi_{r}}^{\xi_{r}} \overline{\boldsymbol{U}} \overline{\boldsymbol{\Phi}}_{x}^{\mathrm{T}} \mathrm{d} \xi \mathrm{d} \theta \cdot I_{P} \\
& \boldsymbol{M}^{v v}=L R \sum_{s=1}^{N P} \int_{\theta_{s}}^{\theta_{s}^{\prime}} \int_{\xi_{s}}^{\xi_{s}^{\prime}} \overline{\boldsymbol{V}} \overline{\boldsymbol{V}}^{\mathrm{T}} \mathrm{d} \xi \mathrm{d} \theta \cdot I_{0}+L R \sum_{r=1}^{\overline{N P}} \int_{\theta_{r}}^{\theta_{r}} \int_{\xi_{r}}^{\xi_{r}} \overline{\boldsymbol{V}} \overline{\boldsymbol{V}}^{\mathrm{T}} \mathrm{d} \xi \mathrm{d} \theta \cdot \mathscr{\%} \\
& \boldsymbol{M}^{v \phi_{\theta}}=2 L R \sum_{s=1}^{N P} \int_{\theta_{s}}^{\theta_{s}^{\prime}} \int_{\xi_{s}}^{\xi_{s}^{\prime}} \overline{\boldsymbol{U}} \overline{\boldsymbol{\Phi}}_{\theta}^{\mathrm{T}} \mathrm{d} \xi \mathrm{d} \theta \cdot I_{1}+2 L R \sum_{r=1}^{\overline{N P}} \int_{\theta_{r}}^{\theta_{r}} \int_{\xi_{r}}^{\xi_{r}} \overline{\boldsymbol{U}} \overline{\boldsymbol{\Phi}}_{\theta}^{\mathrm{T}} \mathrm{d} \xi \mathrm{d} \theta \cdot \mathscr{P} \\
& \boldsymbol{M}^{w w}=L R \sum_{s=1}^{N P} \int_{\theta_{s}}^{\theta_{s}^{\prime}} \int_{\xi_{s}}^{\xi_{s}^{\prime}} \overline{\boldsymbol{W}} \overline{\boldsymbol{W}}^{\mathrm{T}} \mathrm{d} \xi \mathrm{d} \theta \cdot I_{0}+L R \sum_{r=1}^{\overline{N P}} \int_{\theta_{r}^{\circ}}^{\theta_{r}} \int_{\xi_{r}}^{\xi_{r}} \overline{\boldsymbol{W}} \overline{\boldsymbol{W}}^{\mathrm{T}} \mathrm{d} \xi \mathrm{d} \theta \cdot \mathscr{P} \\
& \boldsymbol{M}^{\phi_{x} \phi_{x}}=L R \sum_{s=1}^{N P} \int_{\theta_{s}}^{\theta_{s}^{\prime}} \int_{\xi_{s}}^{\xi_{s}^{\prime}} \overline{\boldsymbol{\Phi}}_{x} \overline{\boldsymbol{\Phi}}_{x}^{\mathrm{T}} \mathrm{d} \xi \mathrm{d} \theta \cdot I_{2}+L R \sum_{r=1}^{\overline{N P}} \int_{\theta_{r}}^{\theta_{r}} \int_{\xi_{r}}^{\xi_{r}} \overline{\boldsymbol{\Phi}}_{x} \overline{\boldsymbol{\Phi}}_{x}^{\mathrm{T}} \mathrm{d} \xi \mathrm{d} \theta \cdot \mathscr{I} / \\
& \boldsymbol{M}^{\phi_{\theta} \phi_{\theta}}=L R \sum_{s=1}^{N P} \int_{\theta_{s}}^{\theta_{s}^{\prime}} \int_{\xi_{s}}^{\xi_{s}^{\prime}} \overline{\boldsymbol{\Phi}}_{\theta} \overline{\boldsymbol{\Phi}}_{\theta}^{\mathrm{T}} \mathrm{d} \xi \mathrm{d} \theta \cdot I_{2}+L R \sum_{r=1}^{\overline{N P}} \int_{\theta_{r}}^{\theta_{r}} \int_{\xi_{r}}^{\xi_{r}} \overline{\boldsymbol{\Phi}_{\theta}} \overline{\boldsymbol{\Phi}}_{\theta}^{\mathrm{T}} \mathrm{d} \xi \mathrm{d} \theta \cdot \mathscr{\%}
\end{aligned}
$$

\section{Appendix B. Expressions of the stiffness matrix $\mathrm{K}$}

3 The generalized stiffness matrix of the piezoelectric laminated shell is expressed as 4

$5 \quad \boldsymbol{K}_{q q}=\left[\begin{array}{lllll}\boldsymbol{K}^{u u} & \frac{1}{2} \boldsymbol{K}^{u v} & \frac{1}{2} \boldsymbol{K}^{u w} & \frac{1}{2} \boldsymbol{K}^{u \phi_{x}} & \frac{1}{2} \boldsymbol{K}^{u \phi_{\theta}} \\ \frac{1}{2} \boldsymbol{K}^{u v \mathrm{~T}} & \boldsymbol{K}^{v v} & \frac{1}{2} \boldsymbol{K}^{v w} & \frac{1}{2} \boldsymbol{K}^{v \phi_{x}} & \frac{1}{2} \boldsymbol{K}^{v \phi_{\theta}} \\ \frac{1}{2} \boldsymbol{K}^{u w \mathrm{~T}} & \frac{1}{2} \boldsymbol{K}^{v w \mathrm{~T}} & \boldsymbol{K}^{w w} & \frac{1}{2} \boldsymbol{K}^{w \phi_{x}} & \frac{1}{2} \boldsymbol{K}^{w \phi_{\theta}} \\ \frac{1}{2} \boldsymbol{K}^{u \phi_{x} \mathrm{~T}} & \frac{1}{2} \boldsymbol{K}^{v \phi_{x} \mathrm{~T}} & \frac{1}{2} \boldsymbol{K}^{w \phi_{x} \mathrm{~T}} & \boldsymbol{K}^{\phi_{x} \phi_{x}} & \frac{1}{2} \boldsymbol{K}^{\phi_{x} \phi_{\theta}} \\ \frac{1}{2} \boldsymbol{K}^{u \phi_{\theta} \mathrm{T}} & \frac{1}{2} \boldsymbol{K}^{v \phi_{\theta} \mathrm{T}} & \frac{1}{2} \boldsymbol{K}^{w \phi_{\theta} \mathrm{T}} & \frac{1}{2} \boldsymbol{K}^{\phi_{x} \phi_{\theta} \mathrm{T}} & \boldsymbol{K}^{\phi_{\theta} \phi_{\theta}}\end{array}\right]$

6 where

$$
\begin{aligned}
\boldsymbol{K}^{u u}= & L R \sum_{s=1}^{N P} \int_{\theta_{s}}^{\theta_{s}^{\prime}} \int_{\xi_{s}}^{\xi_{s}^{\prime}}\left(\frac{A_{11}}{L^{2}} \frac{\partial \overline{\boldsymbol{U}}}{\partial \xi} \frac{\partial \overline{\boldsymbol{U}}^{\mathrm{T}}}{\partial \xi}+\frac{A_{66}}{R^{2}} \frac{\partial \overline{\boldsymbol{U}}}{\partial \theta} \frac{\partial \overline{\boldsymbol{U}}^{\mathrm{T}}}{\partial \theta}\right) \mathrm{d} \xi \mathrm{d} \theta \\
& +L R \sum_{r=1}^{\overline{N P}} \int_{\theta_{r}^{\prime}}^{\theta_{r}^{\prime}} \int_{\xi_{r}}^{\xi_{r}}\left(\frac{A_{11}^{\prime}}{L^{2}} \frac{\partial \overline{\boldsymbol{U}}}{\partial \xi} \frac{\partial \overline{\boldsymbol{U}}^{\mathrm{T}}}{\partial \xi}+\frac{A_{66}^{o}}{R^{2}} \frac{\partial \overline{\boldsymbol{U}}}{\partial \theta} \frac{\partial \overline{\boldsymbol{U}}^{\mathrm{T}}}{\partial \theta}\right) \mathrm{d} \xi \mathrm{d} \theta \\
\boldsymbol{K}^{u v} & =L R \sum_{s=1}^{N P} \int_{\theta_{s}}^{\theta_{s}^{\prime}} \int_{\xi_{s}}^{\xi_{s}^{\prime}}\left(\frac{2 A_{12}}{L R} \frac{\partial \overline{\boldsymbol{U}}}{\partial \xi} \frac{\partial \overline{\boldsymbol{V}}^{\mathrm{T}}}{\partial \theta}+\frac{2 A_{66}}{L R} \frac{\partial \overline{\boldsymbol{U}}}{\partial \theta} \frac{\partial \overline{\boldsymbol{V}}^{\mathrm{T}}}{\partial \xi}\right) \mathrm{d} \xi \mathrm{d} \theta
\end{aligned}
$$




$$
\begin{aligned}
& +L R \sum_{r=1}^{\overline{N P}} \int_{\theta_{r}}^{\theta_{r}} \int_{\xi_{r}}^{\xi_{r}}\left(\frac{2 \AA_{12}^{\circ}}{L R} \frac{\partial \overline{\boldsymbol{U}}}{\partial \xi} \frac{\partial \overline{\boldsymbol{V}}^{\mathrm{T}}}{\partial \theta}+\frac{2 \AA_{60}^{\circ}}{L R} \frac{\partial \overline{\boldsymbol{U}}}{\partial \theta} \frac{\partial \overline{\boldsymbol{V}}^{\mathrm{T}}}{\partial \xi}\right) \mathrm{d} \xi \mathrm{d} \theta \\
& \boldsymbol{K}^{u w}=L R \sum_{s=1}^{N P} \int_{\theta_{s}}^{\theta_{s}^{\prime}} \int_{\xi_{s}}^{\xi_{s}}\left(\frac{2 A_{12}}{L R} \frac{\partial \overline{\boldsymbol{U}}}{\partial \xi} \overline{\boldsymbol{W}}^{\mathrm{T}}\right) \mathrm{d} \xi \mathrm{d} \theta+L R \sum_{r=1}^{\overline{N P}} \int_{\theta_{r}^{\prime}}^{\theta_{r}^{\xi_{r}}} \int_{\xi_{r}}^{\xi_{r}}\left(\frac{2 \AA_{12}^{\prime}}{L R} \frac{\partial \overline{\boldsymbol{U}}}{\partial \xi} \overline{\boldsymbol{W}}^{\mathrm{T}}\right) \mathrm{d} \xi \mathrm{d} \theta \\
& \boldsymbol{K}^{u \phi_{x}}=L R \sum_{s=1}^{N P} \int_{\theta_{s}}^{\theta_{s}^{\prime}} \int_{\xi_{s}}^{\xi_{s}^{\prime}}\left(\frac{2 B_{11}}{L^{2}} \frac{\partial \overline{\boldsymbol{U}}}{\partial \xi} \frac{\partial \overline{\boldsymbol{\Phi}}_{x}^{\mathrm{T}}}{\partial \xi}+\frac{2 B_{66}}{R^{2}} \frac{\partial \overline{\boldsymbol{U}}}{\partial \theta} \frac{\partial \overline{\boldsymbol{\Phi}}_{x}^{\mathrm{T}}}{\partial \theta}\right) \mathrm{d} \xi \mathrm{d} \theta \\
& +L R \sum_{r=1}^{\overline{N P}} \int_{\theta_{r}^{0}}^{\theta_{r}^{b}} \int_{\xi_{r}}^{\xi_{r}}\left(\frac{2 B_{11}^{\circ}}{L^{2}} \frac{\partial \overline{\boldsymbol{U}}}{\partial \xi} \frac{\partial \overline{\boldsymbol{\Phi}}_{x}^{\mathrm{T}}}{\partial \xi}+\frac{2 B_{66}^{o}}{R^{2}} \frac{\partial \overline{\boldsymbol{U}}}{\partial \theta} \frac{\partial \overline{\boldsymbol{\Phi}}_{x}^{\mathrm{T}}}{\partial \theta}\right) \mathrm{d} \xi \mathrm{d} \theta \\
& \boldsymbol{K}^{u \phi_{\theta}}=L R \sum_{s=1}^{N P} \int_{\theta_{s}}^{\theta_{s}^{\prime}} \int_{\xi_{s}}^{\xi_{s}}\left(\frac{2 B_{12}}{L R} \frac{\partial \overline{\boldsymbol{U}}}{\partial \xi} \frac{\partial \overline{\boldsymbol{\Phi}}_{\theta}^{\mathrm{T}}}{\partial \theta}+\frac{2 B_{66}}{L R} \frac{\partial \overline{\boldsymbol{U}}}{\partial \theta} \frac{\partial \overline{\boldsymbol{\Phi}}_{\theta}^{\mathrm{T}}}{\partial \xi}\right) \mathrm{d} \xi \mathrm{d} \theta \\
& +L R \sum_{r=1}^{\overline{N P}} \int_{\theta_{r}^{r}}^{\theta_{r}} \int_{\xi_{r}}^{\xi_{r}}\left(+\frac{2 B_{12}^{\circ}}{L R} \frac{\partial \overline{\boldsymbol{U}}}{\partial \xi} \frac{\partial \overline{\boldsymbol{\Phi}}_{\theta}^{\mathrm{T}}}{\partial \theta}+\frac{2 B_{66}^{\circ}}{L R} \frac{\partial \overline{\boldsymbol{U}}}{\partial \theta} \frac{\partial \overline{\boldsymbol{\Phi}}_{\theta}^{\mathrm{T}}}{\partial \xi}\right) \mathrm{d} \xi \mathrm{d} \theta \\
& \boldsymbol{K}^{v v}=L R \sum_{s=1}^{N P} \int_{\theta_{s}}^{\theta_{s}^{\prime}} \int_{\xi_{s}}^{\xi_{s}^{\prime}}\left(\frac{A_{22}}{R^{2}} \frac{\partial \overline{\boldsymbol{V}}}{\partial \theta} \frac{\partial \overline{\boldsymbol{V}}^{\mathrm{T}}}{\partial \theta}+\frac{k_{c} A_{44}}{R^{2}} \overline{\boldsymbol{V}}^{\mathrm{T}}+\frac{A_{66}}{L^{2}} \frac{\partial \overline{\boldsymbol{V}}}{\partial \xi} \frac{\partial \overline{\boldsymbol{V}}^{\mathrm{T}}}{\partial \xi}\right) \mathrm{d} \xi \mathrm{d} \theta \\
& +L R \sum_{r=1}^{\overline{N P}} \int_{\theta_{r}}^{\theta_{r}} \int_{\xi_{r}}^{\xi_{t}}\left(\frac{\AA_{22}^{o}}{R^{2}} \frac{\partial \overline{\boldsymbol{V}}}{\partial \theta} \frac{\partial \overline{\boldsymbol{V}}^{\mathrm{T}}}{\partial \theta}+\frac{k_{c} \AA_{4}^{o}}{R^{2}} \overline{\boldsymbol{V}} \overline{\boldsymbol{V}}^{\mathrm{T}}+\frac{\AA_{66}^{o}}{L^{2}} \frac{\partial \overline{\boldsymbol{V}}}{\partial \xi} \frac{\partial \overline{\boldsymbol{V}}^{\mathrm{T}}}{\partial \xi}\right) \mathrm{d} \xi \mathrm{d} \theta
\end{aligned}
$$$$
1 \quad \boldsymbol{K}^{v w}=L R \sum_{s=1}^{N P} \int_{\theta_{s}}^{\theta_{s}^{\prime}} \int_{\xi_{s}}^{\xi_{s}^{\prime}}\left(\frac{2 A_{22}}{R^{2}} \frac{\partial \overline{\boldsymbol{V}}}{\partial \theta} \overline{\boldsymbol{W}}^{\mathrm{T}}-\frac{2 k_{c} A_{44}}{R^{2}} \overline{\boldsymbol{V}} \frac{\partial \overline{\boldsymbol{W}}^{\mathrm{T}}}{\partial \theta}\right) \mathrm{d} \xi \mathrm{d} \theta
$$$$
+L R \sum_{r=1}^{\overline{N P}} \int_{\theta_{r}}^{\theta_{r}} \int_{\xi_{r}}^{\xi_{r}}\left(\frac{2 \AA_{22}^{\circ}}{R^{2}} \frac{\partial \overline{\boldsymbol{V}}}{\partial \theta} \overline{\boldsymbol{W}}^{\mathrm{T}}-\frac{2 k_{c} \AA_{44}^{o}}{R^{2}} \overline{\boldsymbol{V}} \frac{\partial \overline{\boldsymbol{W}}^{\mathrm{T}}}{\partial \theta}\right) \mathrm{d} \xi \mathrm{d} \theta
$$$$
\boldsymbol{K}^{v \phi_{x}}=L R \sum_{s=1}^{N P} \int_{\theta_{s}}^{\theta_{s}^{s_{s}}} \int_{\xi_{s}}^{\xi_{s}}\left(\frac{2 B_{12}}{L R} \frac{\partial \overline{\boldsymbol{V}}}{\partial \theta} \frac{\partial \overline{\boldsymbol{\Phi}}_{x}^{\mathrm{T}}}{\partial \xi}+\frac{2 B_{66}}{L R} \frac{\partial \overline{\boldsymbol{V}}}{\partial \xi} \frac{\partial \overline{\boldsymbol{\Phi}}_{x}^{\mathrm{T}}}{\partial \theta}\right) \mathrm{d} \xi \mathrm{d} \theta
$$$$
+L R \sum_{r=1}^{\overline{N P}} \int_{\theta_{r}}^{\theta_{r}^{b}} \int_{\xi_{r}}^{\xi_{r}}\left(\frac{2 B_{12}^{o}}{L R} \frac{\partial \overline{\boldsymbol{V}}}{\partial \theta} \frac{\partial \overline{\boldsymbol{\Phi}}_{x}^{\mathrm{T}}}{\partial \xi}+\frac{2 B_{66}^{\circ}}{L R} \frac{\partial \overline{\boldsymbol{V}}}{\partial \xi} \frac{\partial \overline{\boldsymbol{\Phi}}_{x}^{\mathrm{T}}}{\partial \theta}\right) \mathrm{d} \xi \mathrm{d} \theta
$$$$
\boldsymbol{K}^{v \phi_{\theta}}=L R \sum_{s=1}^{N P} \int_{\theta_{s}}^{\theta_{s}^{\prime}} \int_{\xi_{s}}^{\xi_{s}^{\prime}}\left(\frac{2 B_{22}}{R^{2}} \frac{\partial \overline{\boldsymbol{V}}}{\partial \theta} \frac{\partial \overline{\boldsymbol{\Phi}}_{\theta}^{\mathrm{T}}}{\partial \theta}+\frac{2 B_{66}}{L^{2}} \frac{\partial \overline{\boldsymbol{V}}}{\partial \xi} \frac{\partial \overline{\boldsymbol{\Phi}}_{\theta}^{\mathrm{T}}}{\partial \xi}-\frac{2 k_{c} A_{44}}{R} \overline{\boldsymbol{V}} \overline{\boldsymbol{\Phi}}_{\theta}^{\mathrm{T}}\right) \mathrm{d} \xi \mathrm{d} \theta
$$$$
+L R \sum_{r=1}^{\overline{N P}} \int_{\theta_{r}}^{\theta_{r}^{b}} \int_{\xi_{r}}^{g_{r}}\left(\frac{2 B_{22}^{o}}{R^{2}} \frac{\partial \overline{\boldsymbol{V}}}{\partial \theta} \frac{\partial \overline{\boldsymbol{\Phi}}_{\theta}^{\mathrm{T}}}{\partial \theta}+\frac{2 B_{66}^{\circ}}{L^{2}} \frac{\partial \overline{\boldsymbol{V}}}{\partial \xi} \frac{\partial \overline{\boldsymbol{\Phi}}_{\theta}^{\mathrm{T}}}{\partial \xi}-\frac{2 k_{c} \not_{44}^{o}}{R} \overline{\boldsymbol{V}} \overline{\boldsymbol{\Phi}}_{\theta}^{\mathrm{T}}\right) \mathrm{d} \xi \mathrm{d} \theta
$$$$
\boldsymbol{K}^{w w}=L R \sum_{s=1}^{N P} \int_{\theta_{s}}^{\theta_{s}^{\prime}} \int_{\xi_{s}}^{\xi_{s}^{\prime}}\left(\frac{k_{c} A_{44}}{R^{2}} \frac{\partial \overline{\boldsymbol{W}}}{\partial \theta} \frac{\partial \overline{\boldsymbol{W}}^{\mathrm{T}}}{\partial \theta}+\frac{k_{c} A_{55}}{L^{2}} \frac{\partial \overline{\boldsymbol{W}}}{\partial \xi} \frac{\partial \overline{\boldsymbol{W}}^{\mathrm{T}}}{\partial \xi}+\frac{A_{22}}{R^{2}} \overline{\boldsymbol{W}} \overline{\boldsymbol{W}}^{\mathrm{T}}\right) \mathrm{d} \xi \mathrm{d} \theta
$$$$
+L R \sum_{r=1}^{\overline{N P}} \int_{\theta_{r}}^{\theta_{r}^{b}} \int_{\xi_{i r}}^{\xi_{r}}\left(\frac{k_{c} \AA_{4}^{\prime}}{R^{2}} \frac{\partial \overline{\boldsymbol{W}}}{\partial \theta} \frac{\partial \overline{\boldsymbol{W}}^{\mathrm{T}}}{\partial \theta}+\frac{k_{c} \AA_{55}^{c}}{L^{2}} \frac{\partial \overline{\boldsymbol{W}}}{\partial \xi} \frac{\partial \overline{\boldsymbol{W}}^{\mathrm{T}}}{\partial \xi}+\frac{\AA_{22}^{b}}{R^{2}} \overline{\boldsymbol{W}} \overline{\boldsymbol{W}}^{\mathrm{T}}\right) \mathrm{d} \xi \mathrm{d} \theta
$$$$
\boldsymbol{K}^{w \phi_{x}}=L R \sum_{s=1}^{N P} \int_{\theta_{s}}^{\theta_{s}^{\prime}} \int_{\xi_{s}}^{\xi_{s}^{\prime}}\left(\frac{2 k_{c} A_{55}}{L} \frac{\partial \overline{\boldsymbol{W}}}{\partial \xi} \overline{\boldsymbol{\Phi}}_{x}^{\mathrm{T}}+\frac{2 B_{12}}{L R} \overline{\boldsymbol{W}} \frac{\partial \overline{\boldsymbol{\Phi}}_{x}^{\mathrm{T}}}{\partial \xi}\right) \mathrm{d} \xi \mathrm{d} \theta
$$$$
+L R \sum_{r=1}^{\overline{N P}} \int_{\theta_{r}^{c}}^{\theta_{r}^{\xi_{c}}} \int_{\xi_{r}}^{\xi_{r}}\left(\frac{2 k_{c} \AA_{55}^{c}}{L} \frac{\partial \overline{\boldsymbol{W}}}{\partial \xi} \overline{\boldsymbol{\Phi}}_{x}^{\mathrm{T}}+\frac{2 B_{12}^{o}}{L R} \overline{\boldsymbol{W}} \frac{\partial \overline{\boldsymbol{\Phi}}_{x}^{\mathrm{T}}}{\partial \xi}\right) \mathrm{d} \xi \mathrm{d} \theta
$$ 


$$
\begin{aligned}
& \boldsymbol{K}^{w \phi_{\theta}}=L R \sum_{s=1}^{N P} \int_{\theta_{s}}^{\theta_{s}^{\prime}} \int_{\xi_{s}}^{\xi_{s}^{\prime}}\left(\frac{2 k_{c} A_{44}}{R} \frac{\partial \overline{\boldsymbol{W}}}{\partial \theta} \overline{\boldsymbol{\Phi}}_{\theta}^{\mathrm{T}}+\frac{2 B_{22}}{R^{2}} \overline{\boldsymbol{W}} \frac{\partial \overline{\boldsymbol{\Phi}}_{\theta}^{\mathrm{T}}}{\partial \theta}\right) \mathrm{d} \xi \mathrm{d} \theta \\
& +L R \sum_{r=1}^{\overline{N P}} \int_{\theta_{r}}^{\theta_{r}^{b}} \int_{\xi_{r}}^{\xi_{r}^{c}}\left(\frac{2 k_{c} \AA_{44}^{o}}{R} \frac{\partial \overline{\boldsymbol{W}}}{\partial \theta} \overline{\boldsymbol{\Phi}}_{\theta}^{\mathrm{T}}+\frac{2 B_{22}^{\circ}}{R^{2}} \overline{\boldsymbol{W}} \frac{\partial \overline{\boldsymbol{\Phi}}_{\theta}^{\mathrm{T}}}{\partial \theta}\right) \mathrm{d} \xi \mathrm{d} \theta \\
& \boldsymbol{K}^{\phi_{x} \phi_{x}}=L R \sum_{s=1}^{N P} \int_{\theta_{s}}^{\theta_{s}^{\prime}} \int_{\xi_{s}}^{\xi_{s}^{\prime}}\left(\frac{D_{11}}{L^{2}} \frac{\partial \overline{\boldsymbol{\Phi}}_{x}}{\partial \xi} \frac{\partial \overline{\boldsymbol{\Phi}}_{x}^{\mathrm{T}}}{\partial \xi}+\frac{D_{66}}{R^{2}} \frac{\partial \overline{\boldsymbol{\Phi}}_{x}}{\partial \theta} \frac{\partial \overline{\boldsymbol{\Phi}}_{x}^{\mathrm{T}}}{\partial \theta}+k_{c} A_{55} \overline{\boldsymbol{\Phi}}_{x} \overline{\boldsymbol{\Phi}}_{x}^{\mathrm{T}}\right) \mathrm{d} \xi \mathrm{d} \theta \\
& +L R \sum_{r=1}^{\overline{N P}} \int_{\theta_{r}}^{\theta_{r}^{6}} \int_{\xi_{r}}^{\xi_{r}}\left(\frac{B_{11}^{0}}{L^{2}} \frac{\partial \overline{\boldsymbol{\Phi}}_{x}}{\partial \xi} \frac{\partial \overline{\boldsymbol{\Phi}}_{x}^{\mathrm{T}}}{\partial \xi}+\frac{B_{66}}{R^{2}} \frac{\partial \overline{\boldsymbol{\Phi}}_{x}}{\partial \theta} \frac{\partial \overline{\boldsymbol{\Phi}}_{x}^{\mathrm{T}}}{\partial \theta}+k_{c} \AA_{55} \overline{\boldsymbol{\Phi}}_{x} \overline{\boldsymbol{\Phi}}_{x}^{\mathrm{T}}\right) \mathrm{d} \xi \mathrm{d} \theta \\
& \boldsymbol{K}^{\phi_{x} \phi_{\theta}}=L R \sum_{s=1}^{N P} \int_{\theta_{s}}^{\theta_{s}^{\prime}} \int_{\xi_{s}}^{\xi_{s}^{\prime}}\left(\frac{2 D_{12}}{L R} \frac{\partial \overline{\boldsymbol{\Phi}}_{x}}{\partial \xi} \frac{\partial \overline{\boldsymbol{\Phi}}_{\theta}^{\mathrm{T}}}{\partial \theta}+\frac{2 D_{66}}{L R} \frac{\partial \overline{\boldsymbol{\Phi}}_{x}}{\partial \theta} \frac{\partial \overline{\boldsymbol{\Phi}}_{\theta}^{\mathrm{T}}}{\partial \xi}\right) \mathrm{d} \xi \mathrm{d} \theta \\
& +L R \sum_{r=1}^{\overline{N P}} \int_{\theta_{r}}^{\theta_{r}^{6}} \int_{\xi_{r}}^{\xi_{r}}\left(\frac{2 B_{12}^{0}}{L R} \frac{\partial \overline{\boldsymbol{\Phi}}_{x}}{\partial \xi} \frac{\partial \overline{\boldsymbol{\Phi}}_{\theta}^{\mathrm{T}}}{\partial \theta}+\frac{2 B_{66}^{0}}{L R} \frac{\partial \overline{\boldsymbol{\Phi}}_{x}}{\partial \theta} \frac{\partial \overline{\boldsymbol{\Phi}}_{\theta}^{\mathrm{T}}}{\partial \xi}\right) \mathrm{d} \xi \mathrm{d} \theta \\
& \boldsymbol{K}^{\phi_{\theta} \phi_{\theta}}=L R \sum_{s=1}^{N P} \int_{\theta_{s}}^{\theta_{s}^{\prime}} \int_{\xi_{s}}^{\xi_{s}^{\prime}}\left(\frac{D_{22}}{R^{2}} \frac{\partial \overline{\boldsymbol{\Phi}}_{\theta}}{\partial \theta} \frac{\partial \overline{\boldsymbol{\Phi}}_{\theta}^{\mathrm{T}}}{\partial \theta}+k_{c} A_{44} \overline{\boldsymbol{\Phi}}_{\theta} \overline{\boldsymbol{\Phi}}_{\theta}^{\mathrm{T}}+\frac{D_{66}}{L^{2}} \frac{\partial \overline{\boldsymbol{\Phi}}_{\theta}}{\partial \xi} \frac{\partial \overline{\boldsymbol{\Phi}}_{\theta}^{\mathrm{T}}}{\partial \xi}\right) \mathrm{d} \xi \mathrm{d} \theta \\
& +L R \sum_{r=1}^{\overline{N P}} \int_{\theta_{r}}^{\theta_{r}} \int_{\xi_{r}}^{\xi_{r} r}\left(\frac{B_{22}^{o}}{R^{2}} \frac{\partial \overline{\boldsymbol{\Phi}}_{\theta}}{\partial \theta} \frac{\partial \overline{\boldsymbol{\Phi}}_{\theta}^{\mathrm{T}}}{\partial \theta}+k_{c} \AA_{44}^{o} \overline{\boldsymbol{\Phi}}_{\theta} \overline{\boldsymbol{\Phi}}_{\theta}^{\mathrm{T}}+\frac{B_{66}^{0}}{L^{2}} \frac{\partial \overline{\boldsymbol{\Phi}}_{\theta}}{\partial \xi} \frac{\partial \overline{\boldsymbol{\Phi}}_{\theta}^{\mathrm{T}}}{\partial \xi}\right) \mathrm{d} \xi \mathrm{d} \theta
\end{aligned}
$$

2 The Electromechanical coupling stiffness and the electrical stiffness matrix of the piezoelectric

3 laminated shell is expressed as

$4 \quad \boldsymbol{K}_{q \psi}=\left[\begin{array}{ll}0 & 0 \\ \frac{1}{2} \boldsymbol{K}^{v \psi_{a}} & \frac{1}{2} \boldsymbol{K}^{v \psi_{s}} \\ \frac{1}{2} \boldsymbol{K}^{w \psi_{a}} & \frac{1}{2} \boldsymbol{K}^{w \psi_{s}} \\ \frac{1}{2} \boldsymbol{K}^{\phi_{x} \psi_{a}} & \frac{1}{2} \boldsymbol{K}^{\phi_{x} \psi_{s}} \\ \frac{1}{2} \boldsymbol{K}^{\phi_{\theta} \psi_{a}} & \frac{1}{2} \boldsymbol{K}^{\phi_{\theta} \psi_{s}}\end{array}\right]$

$5 \quad \boldsymbol{K}_{\psi \psi}=\left[\begin{array}{ll}\boldsymbol{K}^{\psi_{a} \psi_{a}} & 0 \\ 0 & \boldsymbol{K}^{\psi_{s} \psi_{s}}\end{array}\right]$

6 where

$$
\boldsymbol{K}^{v \psi_{a}}=L R \sum_{s=1}^{N P} \int_{\theta_{s}}^{\theta_{s}^{\prime}} \int_{\xi_{s}}^{\xi_{s}^{\prime}}\left(\frac{e_{24 e}{ }^{a} h_{a}{ }^{3}}{3 R^{2}} \bar{V} \frac{\partial \overline{\boldsymbol{\Psi}}_{a}^{\mathrm{T}}}{\partial \theta}\right) \mathrm{d} \xi \mathrm{d} \theta
$$

$$
\boldsymbol{K}^{v \psi_{s}}=L R \sum_{s=1}^{N P} \int_{\theta_{s}}^{\theta_{s}^{\prime}} \int_{\xi_{s}}^{\xi_{s}^{\prime}}\left(\frac{e_{24 e}{ }^{s} h_{s}^{3}}{3 R^{2}} \bar{V} \frac{\partial \overline{\boldsymbol{\Psi}}_{s}^{\mathrm{T}}}{\partial \theta}\right) \mathrm{d} \xi \mathrm{d} \theta
$$




$$
\begin{aligned}
\boldsymbol{K}^{w \mu_{a}} & =L R \sum_{s=1}^{N P} \int_{\theta_{s}}^{\theta_{s}^{\prime}} \int_{\xi_{s}}^{\xi_{s}^{\prime}}\left\{-\frac{e_{15 e}{ }^{a} h_{a}{ }^{3}}{3 L^{2}} \frac{\partial \overline{\boldsymbol{W}}}{\partial \xi} \frac{\partial \overline{\boldsymbol{\Psi}}_{a}^{\mathrm{T}}}{\partial \xi}-\frac{e_{24 e}{ }^{a} h_{a}{ }^{3}}{3 R^{2}} \frac{\partial \overline{\boldsymbol{W}}}{\partial \theta} \frac{\partial \overline{\boldsymbol{\Psi}}_{a}^{\mathrm{T}}}{\partial \theta}\right\} \mathrm{d} \xi \mathrm{d} \theta \\
\boldsymbol{K}^{m \mu_{s}} & =L R \sum_{s=1}^{N P} \int_{\theta_{s}}^{\theta_{s}} \int_{\xi_{s}}^{\xi_{s}}\left\{-\frac{e_{15 e}{ }^{s} h_{s}^{3}}{3 L^{2}} \frac{\partial \overline{\boldsymbol{W}}}{\partial \xi} \frac{\partial \overline{\boldsymbol{\Psi}}_{s}^{\mathrm{T}}}{\partial \xi}-\frac{e_{24}{ }^{s} h_{s}^{3}}{3 R^{2}} \frac{\partial \overline{\boldsymbol{W}}}{\partial \theta} \frac{\partial \overline{\boldsymbol{\Psi}}_{s}^{\mathrm{T}}}{\partial \theta}\right\} \mathrm{d} \xi \mathrm{d} \theta
\end{aligned}
$$

$1 \quad \boldsymbol{K}^{\phi_{x} \psi_{a}}=L R \sum_{s=1}^{N P} \int_{\theta_{s}}^{\theta_{s}^{\prime}} \int_{\xi_{s}}^{\xi_{s}}\left\{-\frac{e_{15 e}{ }^{a} h_{a}{ }^{3}}{3 L} \overline{\boldsymbol{\Phi}}_{x} \frac{\partial \overline{\boldsymbol{\Psi}}_{a}^{\mathrm{T}}}{\partial \xi}+\frac{e_{31 e}{ }^{a} h_{a}{ }^{3}}{3 L} \frac{\partial \overline{\boldsymbol{\Phi}}_{x}}{\partial \xi} \overline{\boldsymbol{\Psi}}_{a}^{\mathrm{T}}\right\} \mathrm{d} \xi \mathrm{d} \theta$

$$
\begin{aligned}
\boldsymbol{K}^{\phi_{x} \psi_{s}} & =L R \sum_{s=1}^{N P} \int_{\theta_{s}}^{\theta_{s}} \int_{\xi_{s}}^{\xi_{s}^{\prime}}\left\{-\frac{e_{15 e}{ }^{s} h_{s}^{3}}{3 L} \overline{\boldsymbol{\Phi}}_{x} \frac{\partial \overline{\boldsymbol{\Psi}}_{s}^{\mathrm{T}}}{\partial \xi}+\frac{e_{31 e}{ }^{s} h_{s}{ }^{3}}{3 L} \frac{\partial \overline{\boldsymbol{\Phi}}_{x}}{\partial \xi} \overline{\boldsymbol{\Psi}}_{s}^{\mathrm{T}}\right\} \mathrm{d} \xi \mathrm{d} \theta \\
\boldsymbol{K}^{\phi_{\theta} \psi_{a}} & =L R \sum_{s=1}^{N P} \int_{\theta_{s}}^{\theta_{s}^{s}} \int_{\xi_{s}}^{\xi_{s}^{\prime}}\left\{\frac{e_{32 e}{ }^{a} h_{a}{ }^{3}}{3 R} \frac{\partial \overline{\boldsymbol{\Phi}}_{\theta}}{\partial \theta} \overline{\boldsymbol{\Psi}}_{a}^{\mathrm{T}}-\frac{e_{24 e}{ }^{a} h_{a}{ }^{3}}{3 R} \overline{\boldsymbol{\Phi}}_{\theta} \frac{\partial \overline{\boldsymbol{\Psi}}_{a}^{\mathrm{T}}}{\partial \theta}\right\} \mathrm{d} \xi \mathrm{d} \theta
\end{aligned}
$$$$
\boldsymbol{K}^{\phi_{\phi} \psi_{s}}=L R \sum_{s=1}^{N P} \int_{\theta_{s}}^{\theta_{s}^{\prime}} \int_{\xi_{s}}^{\xi_{s}^{\prime}}\left\{\frac{e_{322}^{s} h_{s}^{3}}{3 R} \frac{\partial \overline{\boldsymbol{\Phi}}_{\theta}}{\partial \theta} \overline{\boldsymbol{\Psi}}_{s}^{\mathrm{T}}-\frac{e_{24 e}{ }^{s} h_{s}^{3}}{3 R} \overline{\boldsymbol{\Phi}}_{\theta} \frac{\partial \overline{\boldsymbol{\Psi}}_{s}^{\mathrm{T}}}{\partial \theta}\right\} \mathrm{d} \xi \mathrm{d} \theta
$$

$2 \quad \boldsymbol{K}^{\psi_{a} \psi_{a}}=L R \sum_{s=1}^{N P} \int_{\theta_{s}}^{\theta_{s}} \int_{\xi_{s}}^{\xi_{s}^{\prime}}\left\{-\frac{\zeta_{11 e}{ }^{a} h_{a}{ }^{5}}{30 L^{2}} \frac{\partial \overline{\boldsymbol{\Psi}}_{a}}{\partial \xi} \frac{\partial \overline{\boldsymbol{\Psi}}_{a}^{\mathrm{T}}}{\partial \xi}-\frac{\zeta_{22 e}{ }^{a} h_{a}{ }^{5}}{30 R^{2}} \frac{\partial \overline{\boldsymbol{\Psi}}_{a}}{\partial \theta} \frac{\partial \overline{\boldsymbol{\Psi}}_{a}^{\mathrm{T}}}{\partial \theta}-\frac{\zeta_{33 e}{ }^{a} h_{a}{ }^{3}}{3} \overline{\boldsymbol{\Psi}}_{a} \overline{\boldsymbol{\Psi}}_{a}^{\mathrm{T}}\right\} \mathrm{d} \xi \mathrm{d} \theta$

$$
\boldsymbol{K}^{\psi_{s} \psi_{s}}=L R \sum_{s=1}^{N P} \int_{\theta_{s}}^{\theta_{s}^{\prime}} \int_{\xi_{s}}^{\xi_{s}^{\prime}}\left\{-\frac{\zeta_{11 e}{ }^{s} h_{s}^{5}}{30 L^{2}} \frac{\partial \overline{\boldsymbol{\Psi}}_{s}}{\partial \xi} \frac{\partial \overline{\boldsymbol{\Psi}}_{s}^{\mathrm{T}}}{\partial \xi}-\frac{\zeta_{22 e}{ }^{s} h_{s}^{5}}{30 R^{2}} \frac{\partial \overline{\boldsymbol{\Psi}}_{s}}{\partial \theta} \frac{\partial \overline{\boldsymbol{\Psi}}_{s}^{\mathrm{T}}}{\partial \theta}-\frac{\zeta_{33 e}{ }^{s} h_{s}^{3}}{3} \overline{\boldsymbol{\Psi}}_{s} \overline{\boldsymbol{\Psi}}_{s}^{\mathrm{T}}\right\} \mathrm{d} \xi \mathrm{d} \theta
$$

\section{Appendix C. Expressions of the spring stiffness matrix Kspr}

$4 \quad$ The spring stiffness matrix of the piezoelectric laminated shell is shown as

$5 \quad \boldsymbol{K}_{s p r}=\left[\begin{array}{lllll}\boldsymbol{K}_{s p r}^{u u} & 0 & 0 & 0 & 0 \\ 0 & \boldsymbol{K}_{s p r}^{v v} & 0 & 0 & 0 \\ 0 & 0 & \boldsymbol{K}_{s p r}^{w w} & 0 & 0 \\ 0 & 0 & 0 & \boldsymbol{K}_{s p r}^{\phi_{p} \phi_{x}} & 0 \\ 0 & 0 & 0 & 0 & \boldsymbol{K}_{s p p r}^{\phi_{p} \phi_{\theta}}\end{array}\right]$

6 where

$$
\begin{aligned}
K_{\mathrm{spr}}^{u u} & =\sum_{p=1}^{\mathrm{NA}}\left(k_{\mathrm{u}, p}^{o} \overline{\boldsymbol{U}}\left(0, \theta_{p}\right) \overline{\boldsymbol{U}}^{\mathrm{T}}\left(0, \theta_{p}\right)+k_{u, p}^{1} \overline{\boldsymbol{U}}\left(0, \theta_{p}\right) \overline{\boldsymbol{U}}^{\mathrm{T}}\left(0, \theta_{p}\right)\right) \\
K_{\mathrm{spr}}^{v v} & =\sum_{p=1}^{\mathrm{NA}}\left(k_{v, p}^{0} \overline{\boldsymbol{V}}\left(0, \theta_{p}\right) \overline{\boldsymbol{V}}^{\mathrm{T}}\left(0, \theta_{p}\right)+k_{v, p}^{1} \overline{\boldsymbol{V}}\left(1, \theta_{p}\right) \overline{\boldsymbol{V}}^{\mathrm{T}}\left(1, \theta_{p}\right)\right) \\
7 \quad K_{\mathrm{spr}}^{w w} & =\sum_{p=1}^{\mathrm{NA}}\left(k_{w, p}^{0} \overline{\boldsymbol{W}}\left(0, \theta_{p}\right) \overline{\boldsymbol{W}}^{\mathrm{T}}\left(0, \theta_{p}\right)+k_{w, p}^{\prime 1} \overline{\boldsymbol{W}}\left(1, \theta_{p}\right) \overline{\boldsymbol{W}}^{\mathrm{T}}\left(1, \theta_{p}\right)\right) \\
K_{\mathrm{spr}}^{\phi_{p} \phi_{x}} & =\sum_{p=1}^{\mathrm{NA}}\left(k_{x, p}^{0} \overline{\boldsymbol{\Phi}}_{x}\left(0, \theta_{p}\right) \overline{\boldsymbol{\Phi}}_{x}^{\mathrm{T}}\left(0, \theta_{p}\right)+k_{x, p}^{1} \overline{\boldsymbol{\Phi}}_{x}\left(1, \theta_{p}\right) \overline{\boldsymbol{\Phi}}_{x}^{\mathrm{T}}\left(1, \theta_{p}\right)\right) \\
K_{\mathrm{spr}}^{\phi_{p} \phi_{\theta}} & =\sum_{p=1}^{\mathrm{NA}}\left(k_{\theta, p}^{0} \overline{\boldsymbol{\Phi}}_{\theta}\left(0, \theta_{p}\right) \overline{\boldsymbol{\Phi}}_{\theta}^{\mathrm{T}}\left(0, \theta_{p}\right)+k_{\theta, p}^{1} \overline{\boldsymbol{\Phi}}_{\theta}\left(1, \theta_{p}\right) \overline{\boldsymbol{\Phi}}_{\theta}^{\mathrm{T}}\left(1, \theta_{p}\right)\right)
\end{aligned}
$$




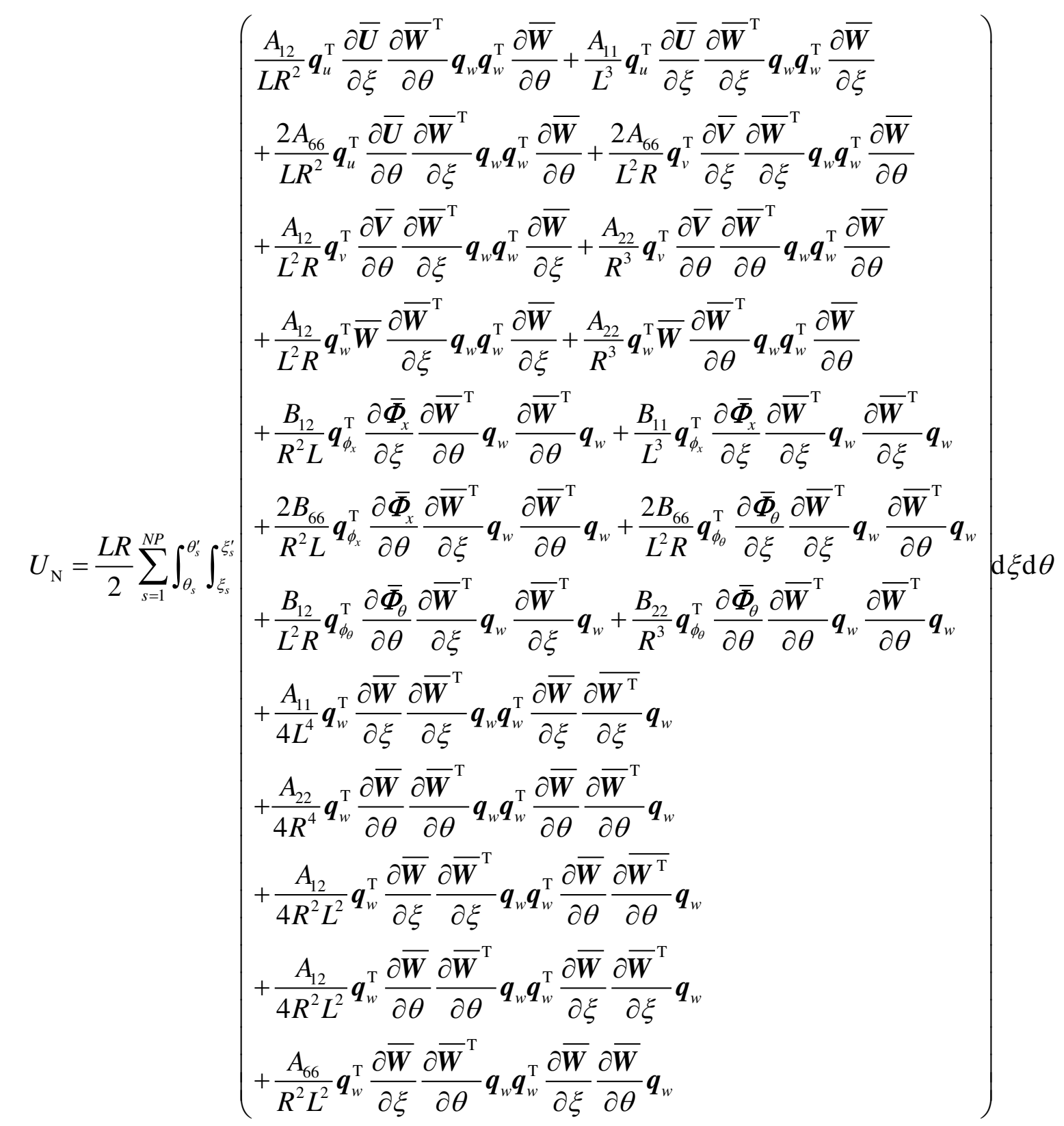

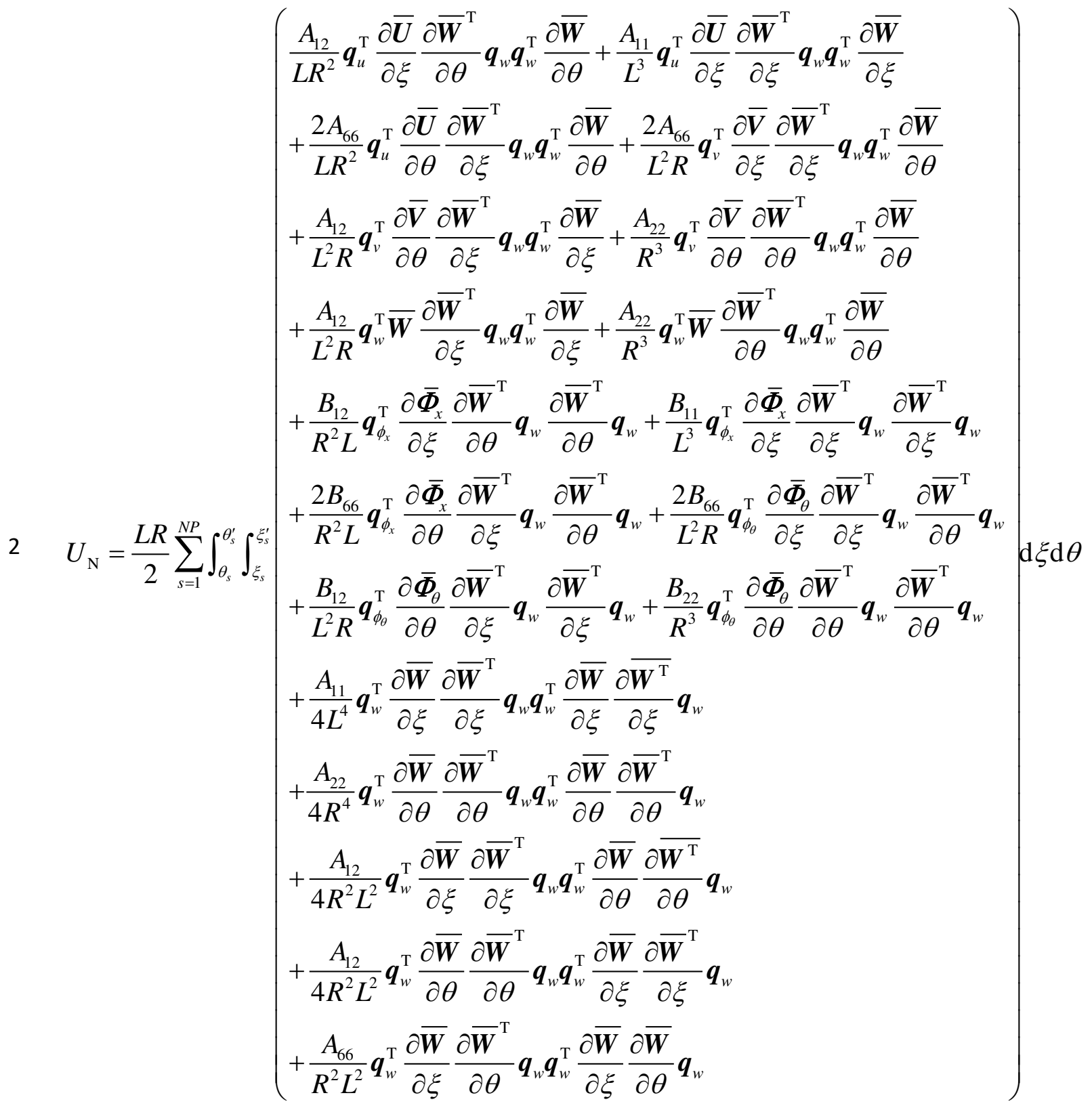




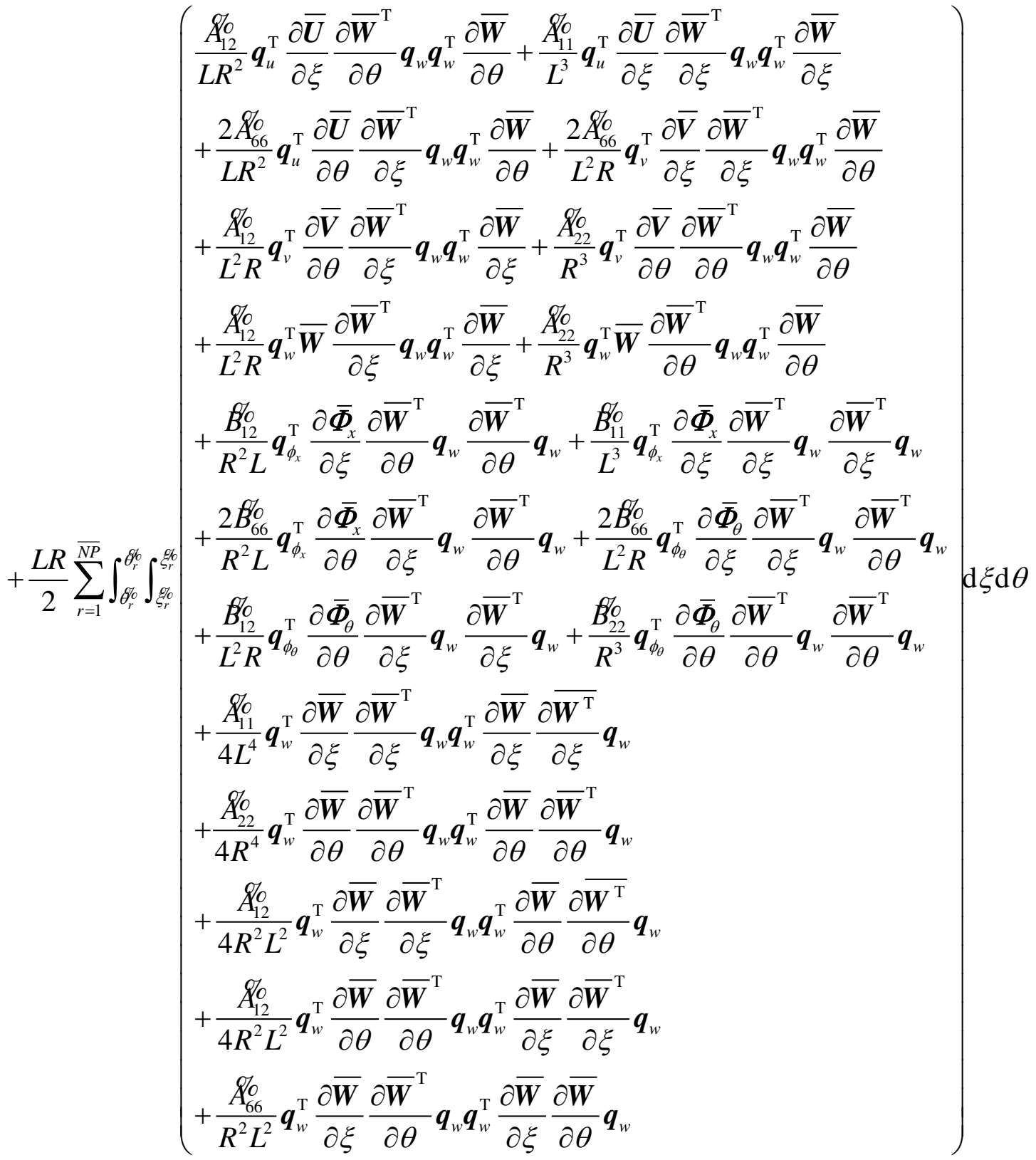

\section{Appendix E. Incremental Harmonic Balance Method}

By presenting the new non-dimensional time variable $\tau$, we write the non-linear vibration differential equation to a modified form $\omega^{2} \boldsymbol{M}_{q q} \boldsymbol{X}^{\prime \prime}+\omega\left(\boldsymbol{C}_{A}+\boldsymbol{C}_{R}\right) \boldsymbol{X}^{\prime}+\left(\boldsymbol{K}_{q q}+\boldsymbol{K}_{s p r}+\boldsymbol{K}_{q \psi}^{S} \boldsymbol{K}_{\psi \psi}^{S}{ }^{-1} \boldsymbol{K}_{q \psi}^{S}{ }^{\mathrm{T}}+\boldsymbol{K}_{\mathrm{N}}^{(2)}+\boldsymbol{K}_{\mathrm{N}}^{(3)}\right) \boldsymbol{X}=\boldsymbol{F} \cos \tau$ $\tau=\omega_{\mathrm{EX}} t, \boldsymbol{X}=\left[X_{1}, X_{2}, X_{3}, \ldots, X_{n}\right]^{\mathrm{T}}, \boldsymbol{F}=\left[\begin{array}{lllll}\boldsymbol{f}_{u} & \boldsymbol{f}_{v} & \boldsymbol{f}_{w} & \boldsymbol{f}_{\phi_{x}} & \boldsymbol{f}_{\phi_{\theta}}\end{array}\right]^{\mathrm{T}}$

Firstly, the differential equations are linearized by using the Newton-Raphson procedure. The corresponding adding increments are used to represent the neighboring state as 
Taking Eq. (D.2) to Eq.(D.1), the higher order terms are neglected, and Eq. (D.1) is written as

3

$$
\omega_{0}^{2} \boldsymbol{M}_{q q} \boldsymbol{X}^{\prime \prime}+\omega_{0}\left(\boldsymbol{C}_{A}+\boldsymbol{C}_{R}\right) \boldsymbol{X}^{\prime}+\left(\boldsymbol{K}_{q q}+\boldsymbol{K}_{s p r}+\boldsymbol{K}_{q \psi}^{S} \boldsymbol{K}_{\psi \psi}^{S}{ }^{-1} \boldsymbol{K}_{q \psi}^{S}{ }^{\mathrm{T}}+2 \boldsymbol{K}_{\mathrm{N}}^{(2)}+3 \boldsymbol{K}_{\mathrm{N}}^{(3)}\right) \boldsymbol{X}
$$$$
=\mathbf{R e}-\left(2 \omega_{0} \boldsymbol{M}_{q q} \boldsymbol{X}_{0}^{\prime \prime}+\left(\boldsymbol{C}_{A}+\boldsymbol{C}_{R}\right) \boldsymbol{X}_{0}^{\prime}\right) \Delta \omega
$$

4

$$
\boldsymbol{R e}=\boldsymbol{F} \cos \tau-\omega_{0}^{2} \boldsymbol{M}_{q q} \boldsymbol{X}_{0}^{\prime \prime}-\omega_{0}\left(\boldsymbol{C}_{A}+\boldsymbol{C}_{R}\right) \boldsymbol{X}_{0}^{\prime}-\left(\boldsymbol{K}_{q q}+\boldsymbol{K}_{q \psi}^{S} \boldsymbol{K}_{\psi \psi}^{S-1} \boldsymbol{K}_{q \psi}^{S \mathrm{~T}}+2 \boldsymbol{K}_{\mathrm{N}}^{(2)}+3 \boldsymbol{K}_{\mathrm{N}}^{(3)}\right) \boldsymbol{X}_{0}
$$

5 where $\boldsymbol{X}_{0}=\left[X_{10}, X_{20}, X_{30}, \ldots, X_{n 0}\right]^{\mathrm{T}}, \Delta \boldsymbol{X}=\left[\Delta X_{1}, \Delta X_{2}, \Delta X_{3}, \ldots, \Delta X_{n}\right]^{\mathrm{T}}$. When the solution is the exact

6 value, the residue "Re" becomes zero.

8

9

$$
X_{j 0}=A_{0}+\sum_{k=1}^{r}\left(a_{j k} \cos k \tau+b_{j k} \sin k \tau\right)=\boldsymbol{T}_{c} \boldsymbol{A}_{j}
$$$$
\Delta X_{j}=\Delta A+\sum_{k=1}^{r}\left(a_{j k} \cos k \tau+b_{j k} \sin k \tau\right)=\boldsymbol{T}_{c} \Delta \boldsymbol{A}_{j}
$$

10

where

$\boldsymbol{T}_{c}=[1, \cos \tau, \sin \tau, \cos 2 \tau, \sin 2 \tau, \mathrm{L}, \cos r \tau, \sin r \tau]$

11

$\boldsymbol{A}_{j}=\left\{a_{j 1}, a_{j 2}, \ldots, a_{j n}, b_{j 1}, b_{j 2}, \ldots, b_{j n}\right\}^{\mathrm{T}}$

$$
\Delta A_{j}=\left\{\Delta a_{j 1}, \Delta a_{j 2}, \ldots, \Delta a_{j n}, \Delta b_{j 1}, \Delta b_{j 2}, \ldots, \Delta b_{j n}\right\}^{\mathrm{T}}
$$

where $a_{j k}, b_{j k}$ are the Fourier coefficients, and $n$ are the numbers of cosine and sine terms. Using these vectors of Fourier coefficients $\boldsymbol{A}$ and its increments $\Delta \boldsymbol{A}$, the vectors of the unknown

$$
X_{0}=S A
$$

$$
\Delta X=S \Delta A
$$


$1 \quad \boldsymbol{S}=\left[\begin{array}{cccc}\boldsymbol{T}_{c} & & & \\ & \boldsymbol{T}_{c} & \\ & & \mathrm{O} & \\ & & & \boldsymbol{T}_{c}\end{array}\right], \boldsymbol{A}=\left\{\boldsymbol{A}_{1}, \boldsymbol{A}_{2}, \ldots, \boldsymbol{A}_{n}\right\}^{\mathrm{T}}, \Delta \boldsymbol{A}=\left\{\Delta \boldsymbol{A}_{1}, \Delta \boldsymbol{A}_{2}, \ldots, \Delta \boldsymbol{A}_{n}\right\}^{\mathrm{T}}$

Taking the Eqs. (D.7) to Eq. (D.3), Galerkin's technique is used

3

$\int_{0}^{2 \pi} \delta(\Delta \boldsymbol{X})^{\mathrm{T}}\left[\omega_{0}^{2} \boldsymbol{M}_{q q} \boldsymbol{X}^{\prime \prime}+\omega_{0}\left(\boldsymbol{C}_{A}+\boldsymbol{C}_{R}\right) \boldsymbol{X}^{\prime}+\left(\boldsymbol{K}_{q q}+\boldsymbol{K}_{s p r}+\boldsymbol{K}_{q \psi}^{S} \boldsymbol{K}_{\psi \psi}^{S-1} \boldsymbol{K}_{q \psi}^{S \mathrm{~T}}+2 \boldsymbol{K}_{\mathrm{N}}^{(2)}+3 \boldsymbol{K}_{\mathrm{N}}^{(3)}\right) \Delta \boldsymbol{X}\right] \mathrm{d} \tau$

$=\int_{0}^{2 \pi} \delta(\Delta \boldsymbol{X})^{\mathrm{T}}\left[\boldsymbol{R e}-\left(2 \omega_{0} \boldsymbol{M}_{q q} \boldsymbol{X}_{0}^{\prime \prime}\right) \Delta \omega-\left(\boldsymbol{C}_{A}+\boldsymbol{C}_{R}\right) \boldsymbol{X}_{0}^{\prime}\right] \mathrm{d} \tau$

The linear equation including $\Delta \boldsymbol{A}$ and $\Delta \omega$ is written as

$\boldsymbol{K}_{\mathrm{mc}} \Delta \boldsymbol{A}+\boldsymbol{R}_{\mathrm{mc}} \boldsymbol{A} \Delta \omega=\boldsymbol{R}_{\mathrm{m} 1} \boldsymbol{A}+\boldsymbol{R}_{\mathrm{m} 2}$

where

$$
\begin{aligned}
& \boldsymbol{K}_{\mathrm{mc}}=\int_{0}^{2 \pi} \boldsymbol{S}^{\mathrm{T}}\left[\omega^{2} \boldsymbol{M}_{q q} \boldsymbol{S}_{+} \omega\left(\boldsymbol{C}_{A}+\boldsymbol{C}_{R}\right) \boldsymbol{S}^{\&}\left(\boldsymbol{K}_{q q}+\boldsymbol{K}_{s p r}+\boldsymbol{K}_{q \psi}^{S} \boldsymbol{K}_{\psi \psi}^{S-1} \boldsymbol{K}_{q \psi}^{S \mathrm{~T}}+2 \boldsymbol{K}_{\mathrm{N}}^{(2)}+3 \boldsymbol{K}_{\mathrm{N}}^{(3)}\right) \boldsymbol{S}\right] \mathrm{d} \tau \\
& \left.\boldsymbol{R}_{\mathrm{mc}}=\int_{0}^{2 \pi} \boldsymbol{S}^{\mathrm{T}}\left(2 \omega \boldsymbol{M}_{q q} \boldsymbol{C}_{A}+\boldsymbol{C}_{R}\right) \boldsymbol{S}^{\ell}\right) \mathrm{d} \tau \\
& \boldsymbol{R}_{\mathrm{m} 1}=-\int_{0}^{2 \pi} \boldsymbol{S}^{\mathrm{T}}\left[\omega^{2} \boldsymbol{M}_{q q} \boldsymbol{C}_{+}\left(\boldsymbol{C}_{A}+\boldsymbol{C}_{R}\right) \boldsymbol{S}^{\&}\left(\boldsymbol{K}_{q q}+\boldsymbol{K}_{s p r}+\boldsymbol{K}_{q \psi}^{S} \boldsymbol{K}_{\psi \psi}^{S-1} \boldsymbol{K}_{q \psi}^{S \mathrm{~T}}+\boldsymbol{K}_{\mathrm{N}}^{(2)}+\boldsymbol{K}_{\mathrm{N}}^{(3)}\right) \boldsymbol{S}\right] \mathrm{d} \tau \\
& \boldsymbol{R}_{\mathrm{m} 2}=\int_{0}^{2 \pi} \boldsymbol{S}^{\mathrm{T}} \boldsymbol{F} \cos \tau \mathrm{d} \tau
\end{aligned}
$$

Finally, we use the arc-length method to solve Eq. (D.10) for obtaining the frequency-amplitude

response.

\section{Conflict of interests}

The authors declare that there is no conflict of interests regarding the publication of this article.

\section{References}

[1] Tzou H S, Gadre M. Theoretical analysis of a multi-layered thin shell coupled with piezoelectric shell actuators for distributed vibration controls. Journal of Sound \& Vibration. 1989;132:433-50.

[2] Tzou H S. Piezoelectric Shells. Solid Mechanics \& Its Applications. 1993;50:98-105.

[3] Kerur S B, Ghosh A. Active vibration control of composite plate using afc actuator and pvdf sensor. International Journal of Structural Stability \& Dynamics. 2011;11:237-55.

[4] Parashar S K, Kumar A. Three-dimensional analytical modeling of vibration behavior of piezoceramic cylindrical shells. Archive of Applied Mechanics. 2015;85:641-56.

[5] Sheng G G, Wang X. Studies on dynamic behavior of functionally graded cylindrical shells with PZT layers under moving loads. Journal of Sound and Vibration. 2009;323:772-89.

[6] Qin Z Y, Chu F L, Zu J. Free vibrations of cylindrical shells with arbitrary boundary conditions: A comparison study. International Journal of Mechanical Sciences. 2017;133:91-9.

[7] Qin Z Y, Pang X J, Safaei B, Chu F L. Free vibration analysis of rotating functionally graded CNT reinforced 
[8] Li C F, Li P Y, Zhang Z X, Wen B C. Optimal locations of discontinuous piezoelectric laminated cylindrical shell with point supported elastic boundary conditions for vibration control. Composite Structures. 2019:111575.

[9] Amabili M. Nonlinear vibrations of laminated circular cylindrical shells: Comparison of different shell theories. Composite Structures. 2011;94:207-20.

[10] Jansen E L. The effect of static loading and imperfections on the nonlinear vibrations of laminated cylindrical shells. Journal of Sound and Vibration. 2008;315:1035-46.

[11] Przekop A, Phairoh T, Huang JK, Mei C. Adaptive Control of Nonlinear Free Vibration of Shallow Shell Using Piezoelectric Actuators. AIAA Journal. 2011;49:472-88.

[12] Rafiee M, Mohammadi M, Aragh BS, Yaghoobi H. Nonlinear free and forced thermo-electro-aero-elastic vibration and dynamic response of piezoelectric functionally graded laminated composite shells : Part II: Numerical results. Composite Structures. 2013;103:188-96.

[13] Rafiee M, Mohammadi M, Aragh B S, Yaghoobi H. Nonlinear free and forced thermo-electro-aero-elastic vibration and dynamic response of piezoelectric functionally graded laminated composite shells, Part I: Theory and analytical solutions. Composite Structures. 2013;103:179-87.

[14] Sheng G G, Wang X. Nonlinear vibration control of functionally graded laminated cylindrical shells. Composites Part B Engineering. 2013;52:1-10.

[15] Shen H S, Yang D Q. Nonlinear vibration of anisotropic laminated cylindrical shells with piezoelectric fiber reinforced composite actuators. Ocean Engineering. 2014;80:36-49.

[16] Zhang S Q, Li Y X, Schmidt R. Active shape and vibration control for piezoelectric bonded composite structures using various geometric nonlinearities. Composite Structures. 2015;122:239-49.

[17] Yue H H, Lu Y F, Deng Z Q, Tzou H. Experiments on vibration control of a piezoelectric laminated paraboloidal shell. Mechanical Systems \& Signal Processing. 2017;82:279-95.

[18] Ninh D G, Bich D H. Characteristics of nonlinear vibration of nanocomposite cylindrical shells with piezoelectric actuators under thermo-mechanical loads. Aerospace Science \& Technology. 2018:S127096381830381X.

[19] Li C F, Li P Y, Zhong B F, Wen B C. Geometrically nonlinear vibration of laminated composite cylindrical thin shells with non-continuous elastic boundary conditions. Nonlinear Dynamics. 2019;95:1903-21.

[20] Li C F, Miao B Q, Tang Q S, Xi C Y, Wen B C. Nonlinear vibrations analysis of rotating drum-disk coupling structure. Journal of Sound \& Vibration. 2018;420:35-60.

[21] Chen Y H, Jin G Y, Liu Z G. Free vibration analysis of circular cylindrical shell with non-uniform elastic boundary constraints. International Journal of Mechanical Sciences. 2013;74:120-32.

[22] Xie K, Chen M X, Zhang L, Xie D. Free and forced vibration analysis of non-uniformly supported cylindrical shells through wave based method. International Journal of Mechanical Sciences. 2017;128:512-26.

[23] Tang Q S, Li C F, Wen B C. Analysis on Forced Vibration of Thin-Wall Cylindrical Shell with Nonlinear Boundary Condition. Shock and Vibration. 2016:22.

[24] Tang Q S, Li C, She H X, Wen B C. Modeling and dynamic analysis of bolted joined cylindrical shell. Nonlinear Dynamics. 2018;93:1953-75.

[25] Li P Y, Li C F, Qiao R H, Wen B C. Sensitivity on the non-continuous supported laminated cylindrical shell to boundary conditions and lamination schemes. Archive of Applied Mechanics. 2019;89:2245-64.

[26] Zhai J J, Zhao G Z, Shang L Y. Integrated design optimization of structural size and control system of piezoelectric curved shells with respect to sound radiation. Structural and Multidisciplinary Optimization. 2017;56:1287-304.

[27] To C W S, Chen T. Optimal control of random vibration in plate and shell structures with distributed piezoelectric components. International Journal of Mechanical Sciences. 2007;49:1389-98. 
Figures

(a)

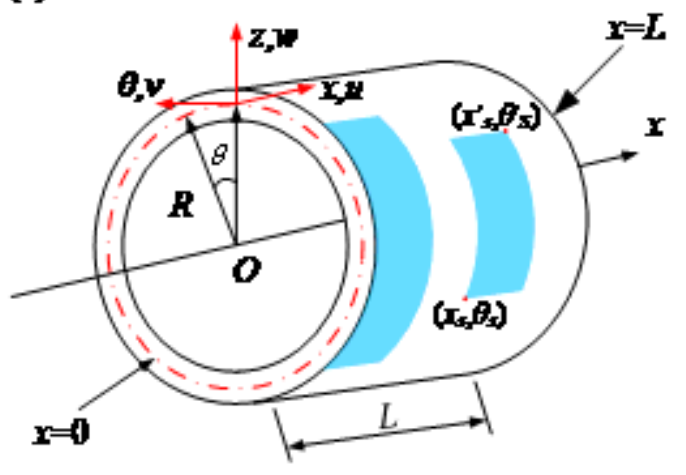

()

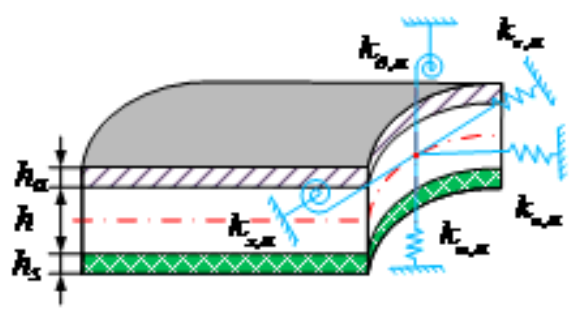

\section{Figure 1}

Schematic diagram of a piezoelectric laminated composite cylindrical shell with elastic boundary conditions: (a) coordinate system and geometry of the shell; (b) partial cross-sectional view of the shell with elastic boundary condition

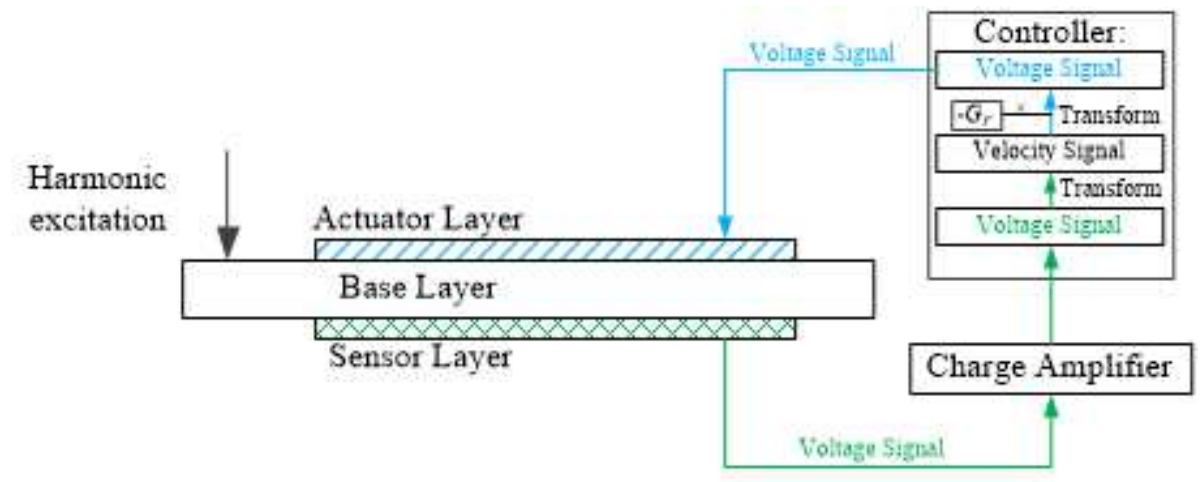

Figure 2

The schematic diagram of feedback control 


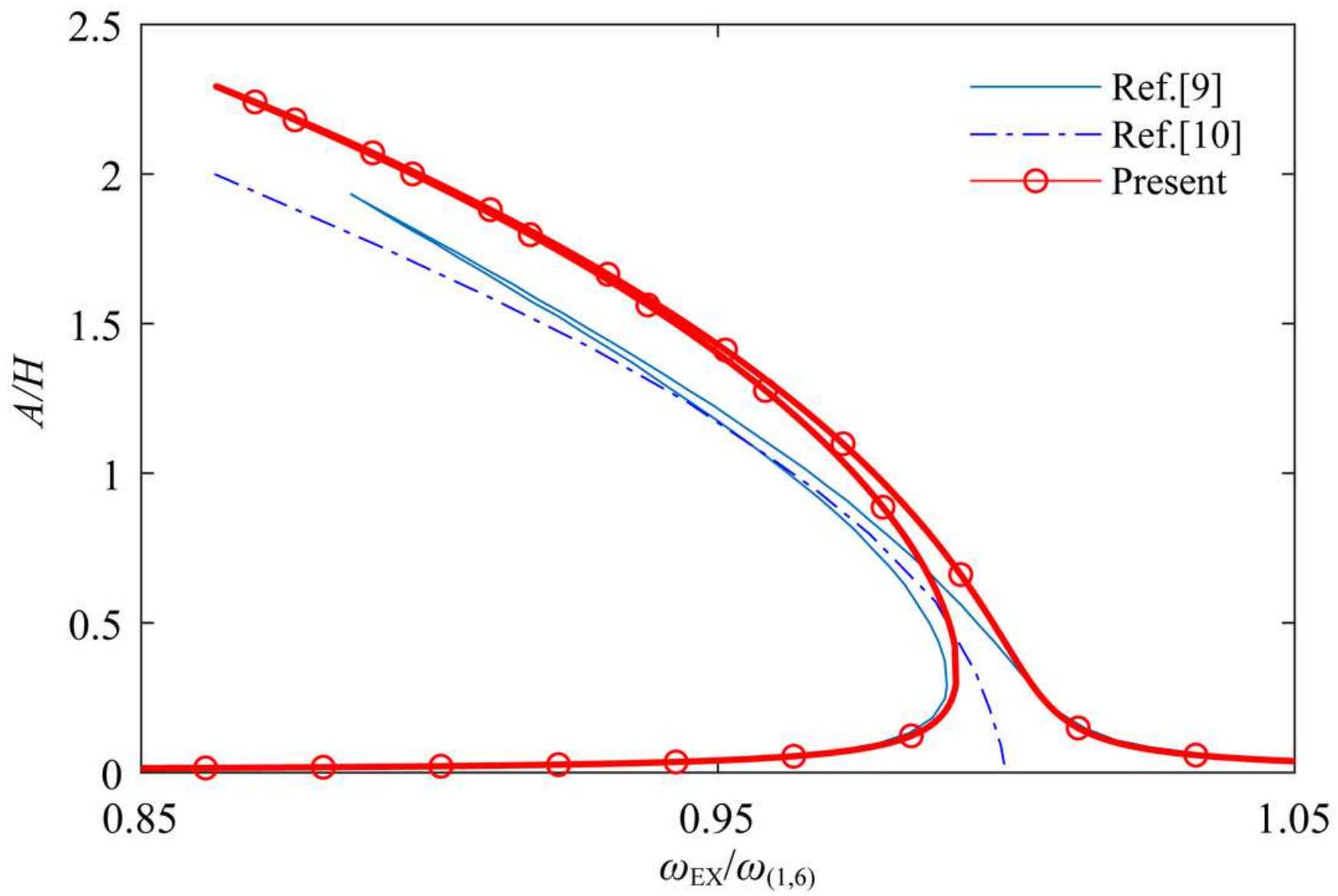

Figure 3

Comparison of amplitude-frequency curves with the references 


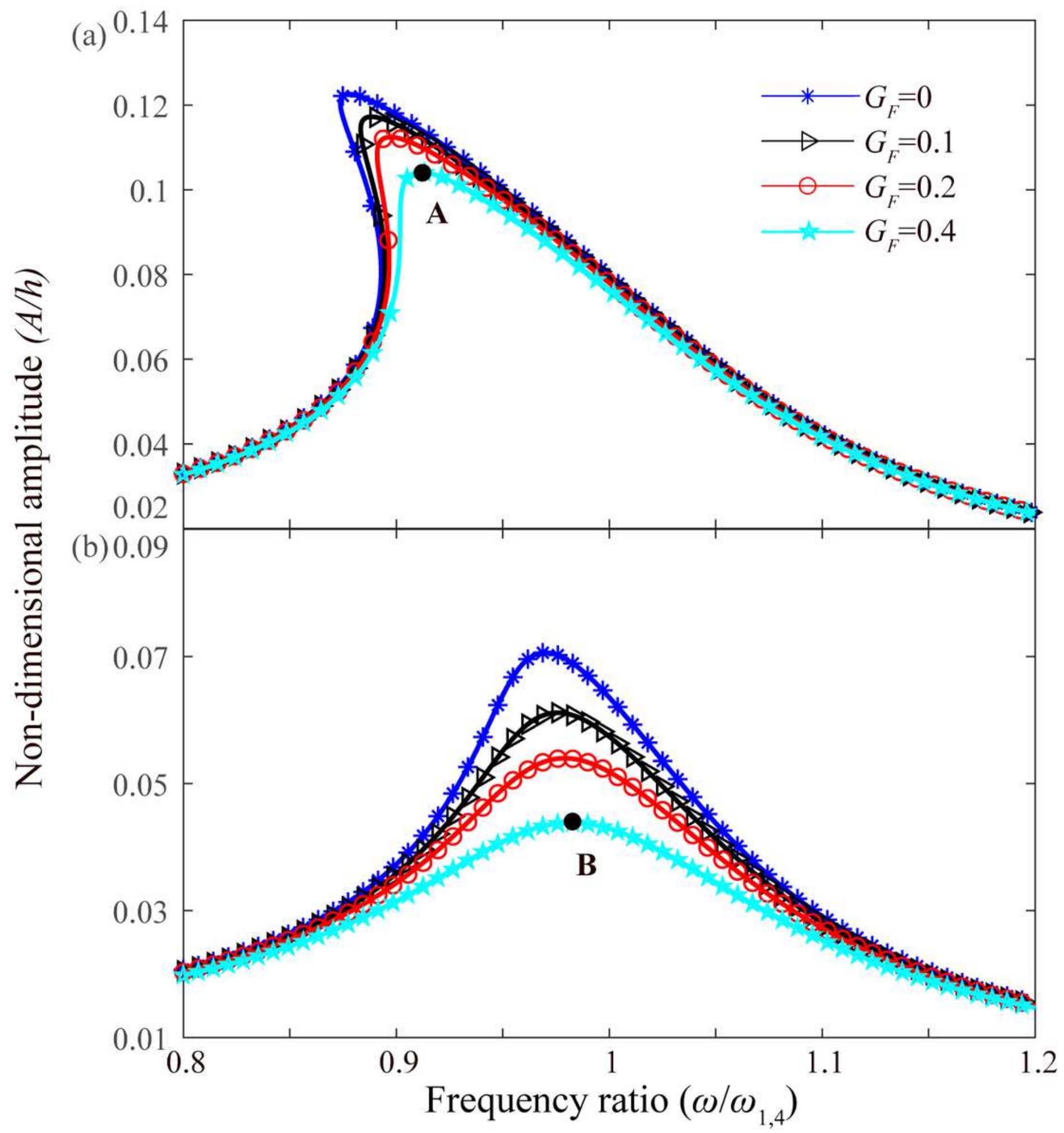

Figure 4

The influence of the constant gain for frequency-amplitude response 


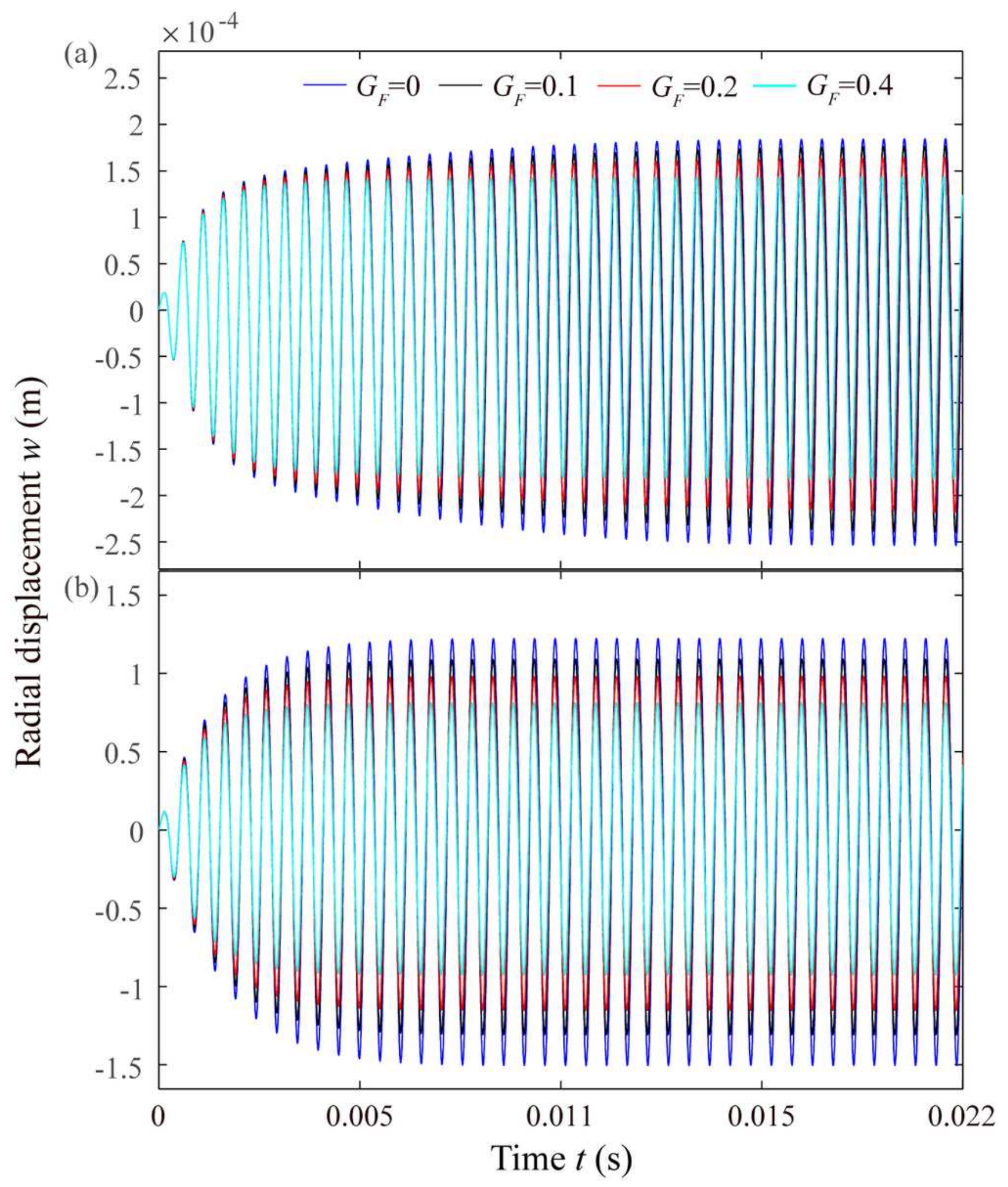

Figure 5

The influence of the constant gain for time-domain response 


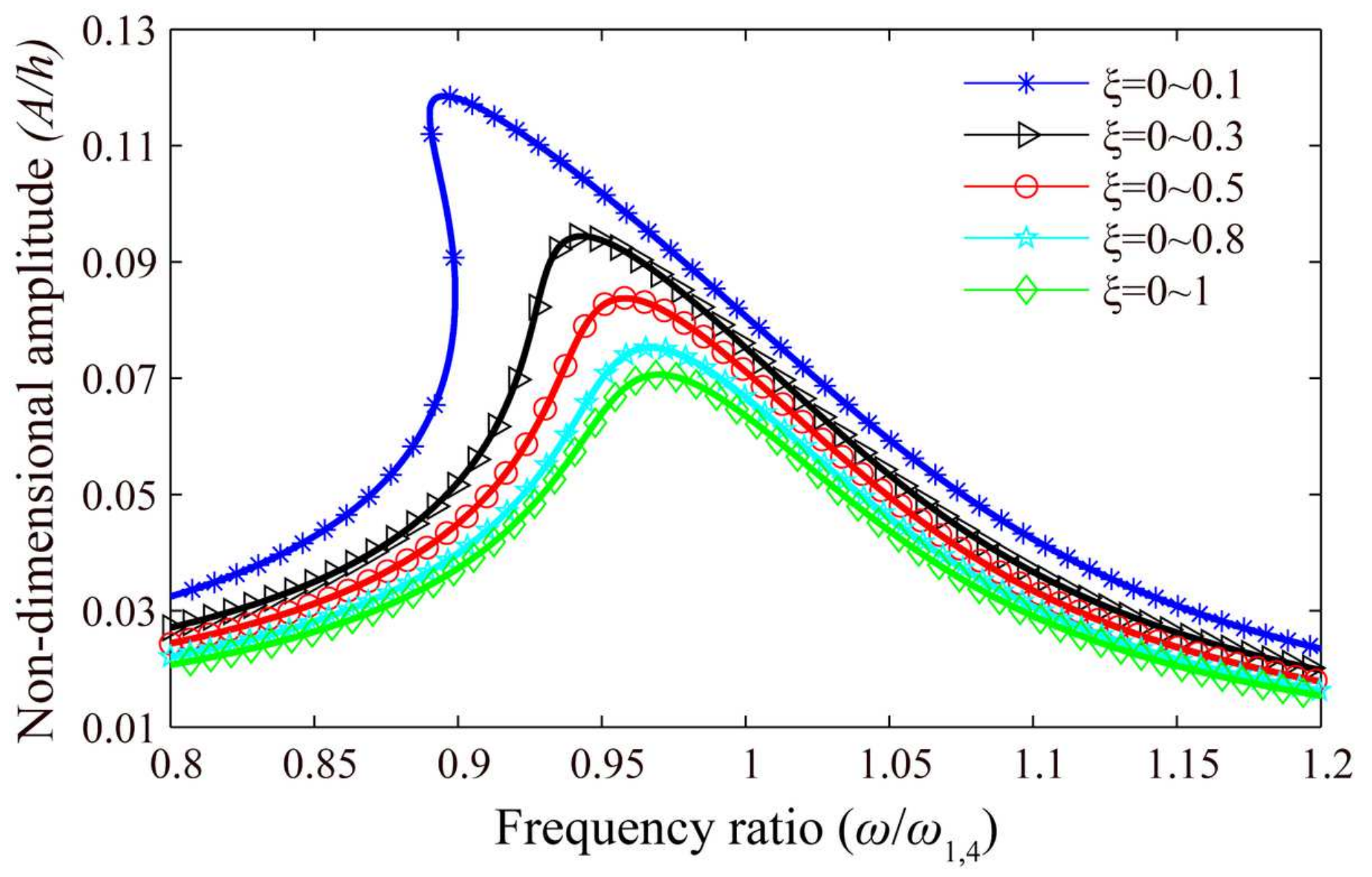

Figure 6

The influence of the size of the piezoelectric layer for frequency-amplitude response 


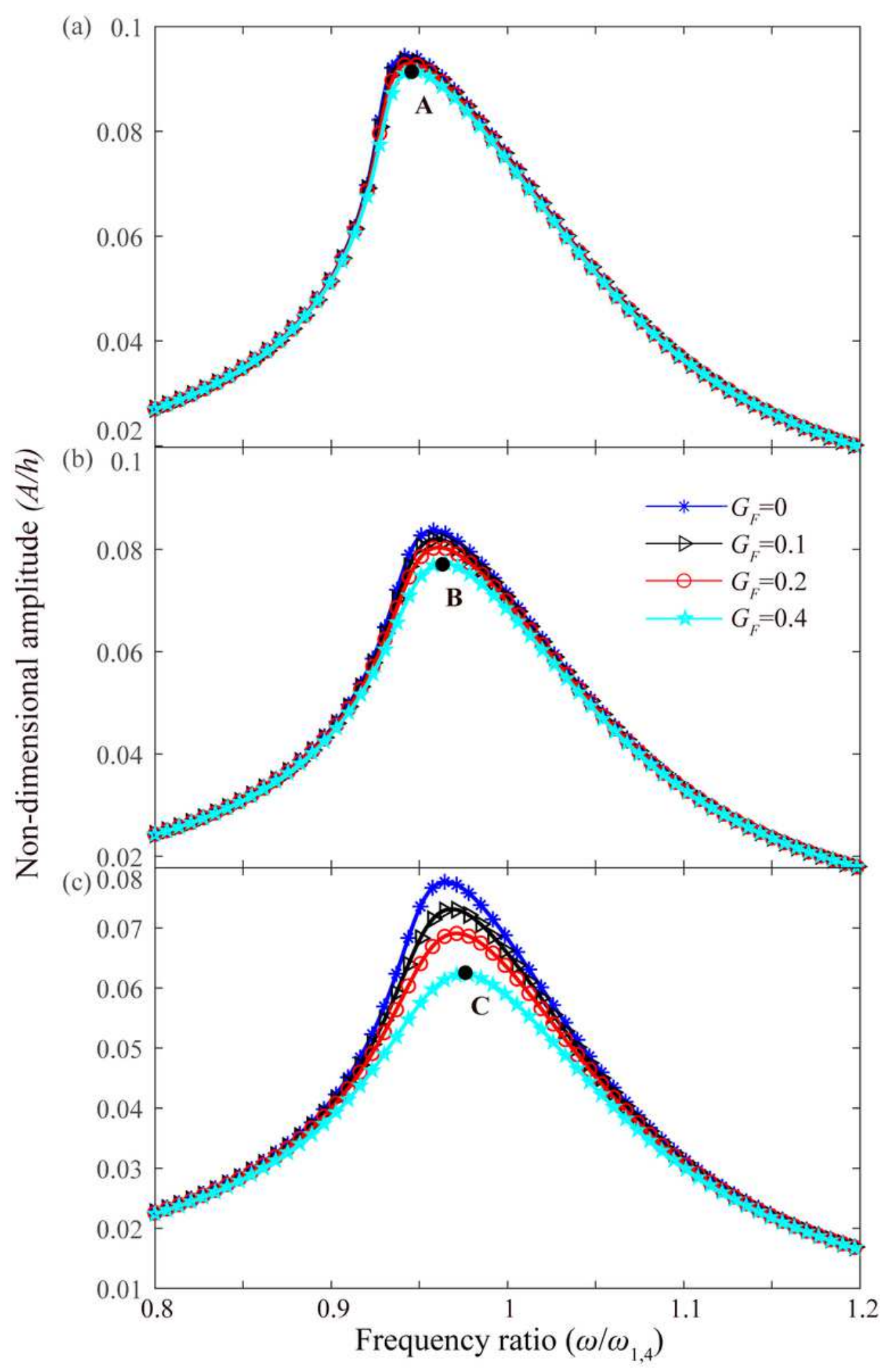

Figure 7

The influences of the size of the piezoelectric layer and constant gain for frequency-amplitude response 


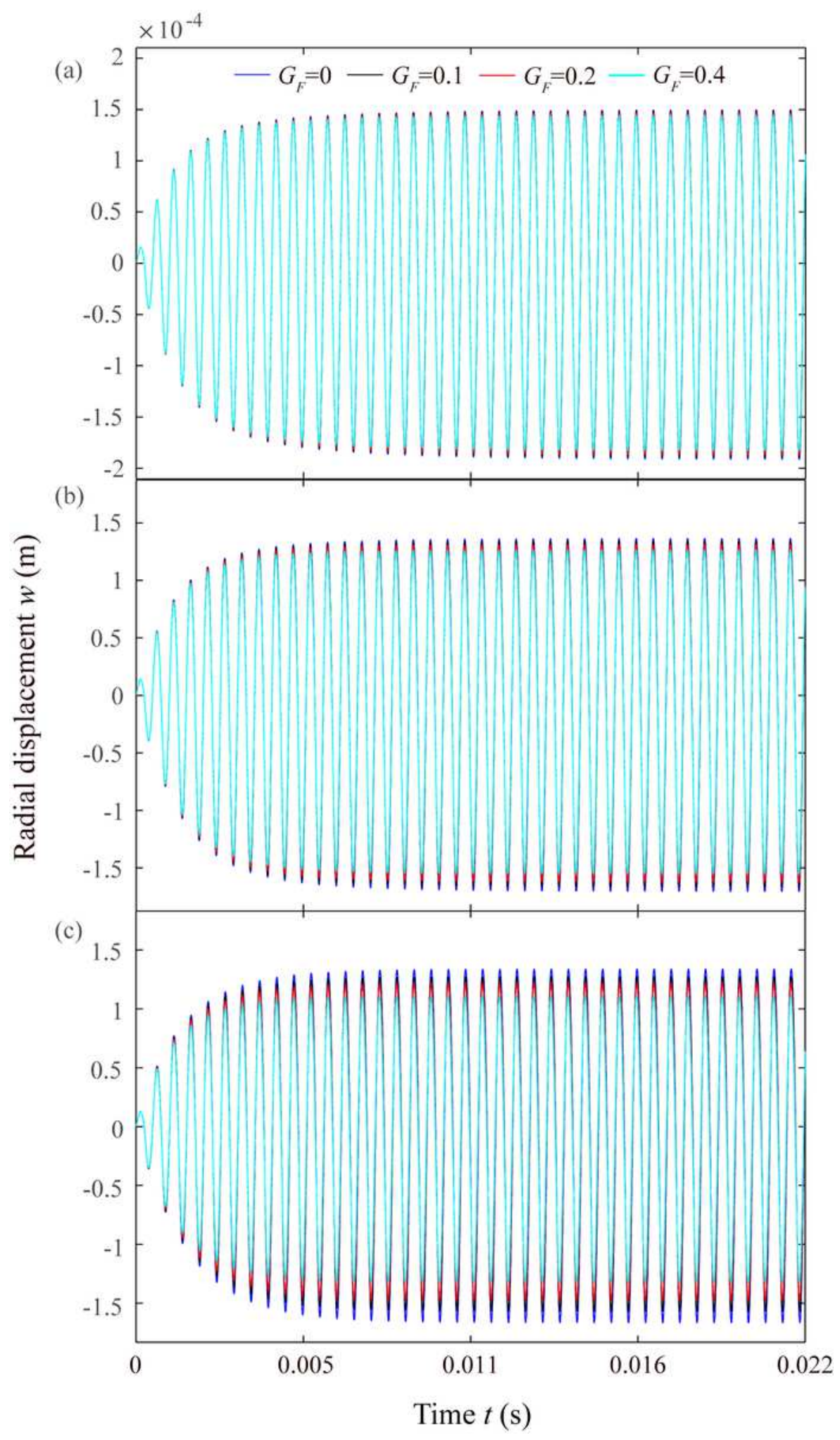

Figure 8

The influences of the size of the piezoelectric layer and constant gain for time-domain response 


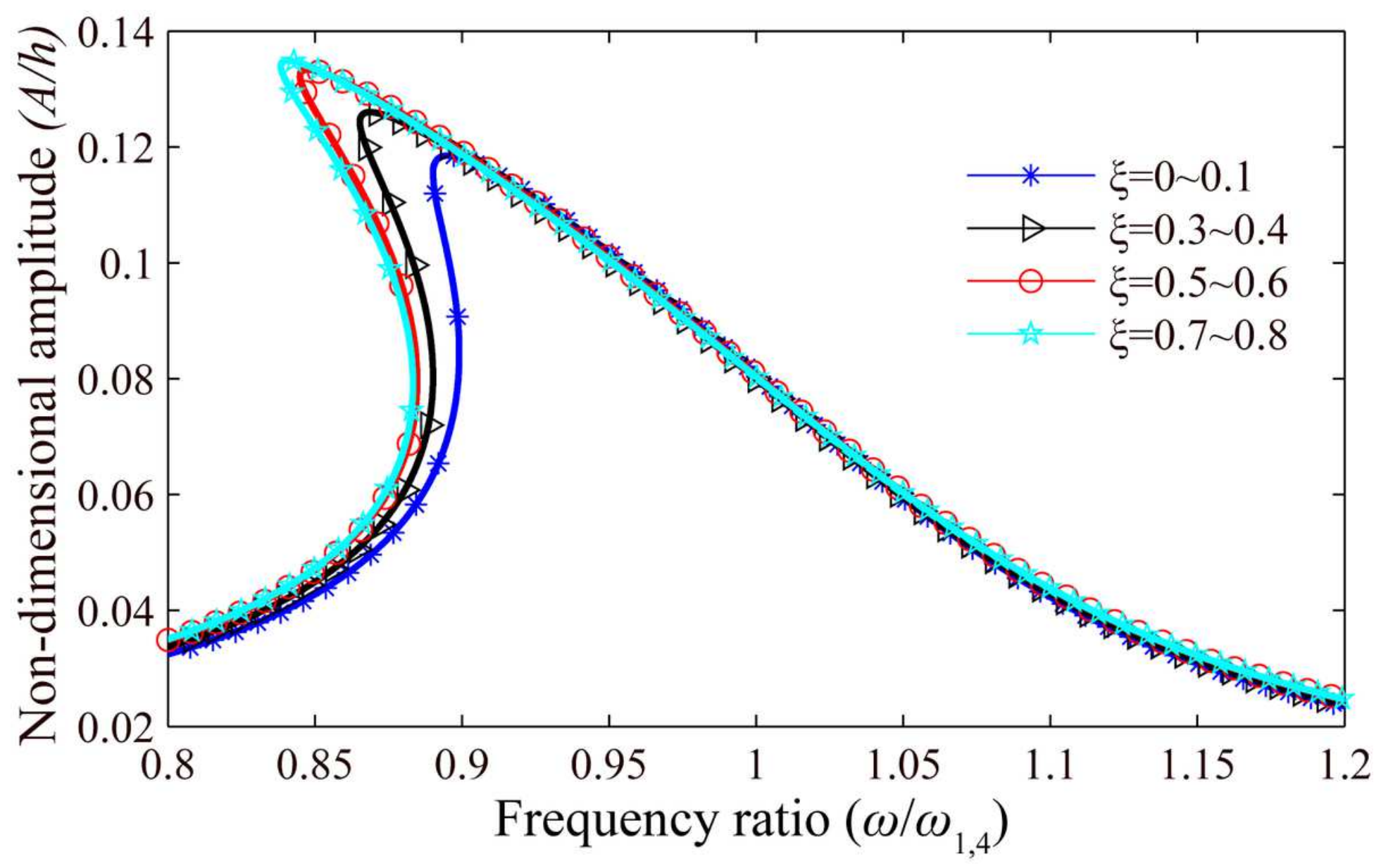

Figure 9

The influence of the position of the piezoelectric layer for frequency-amplitude respon 


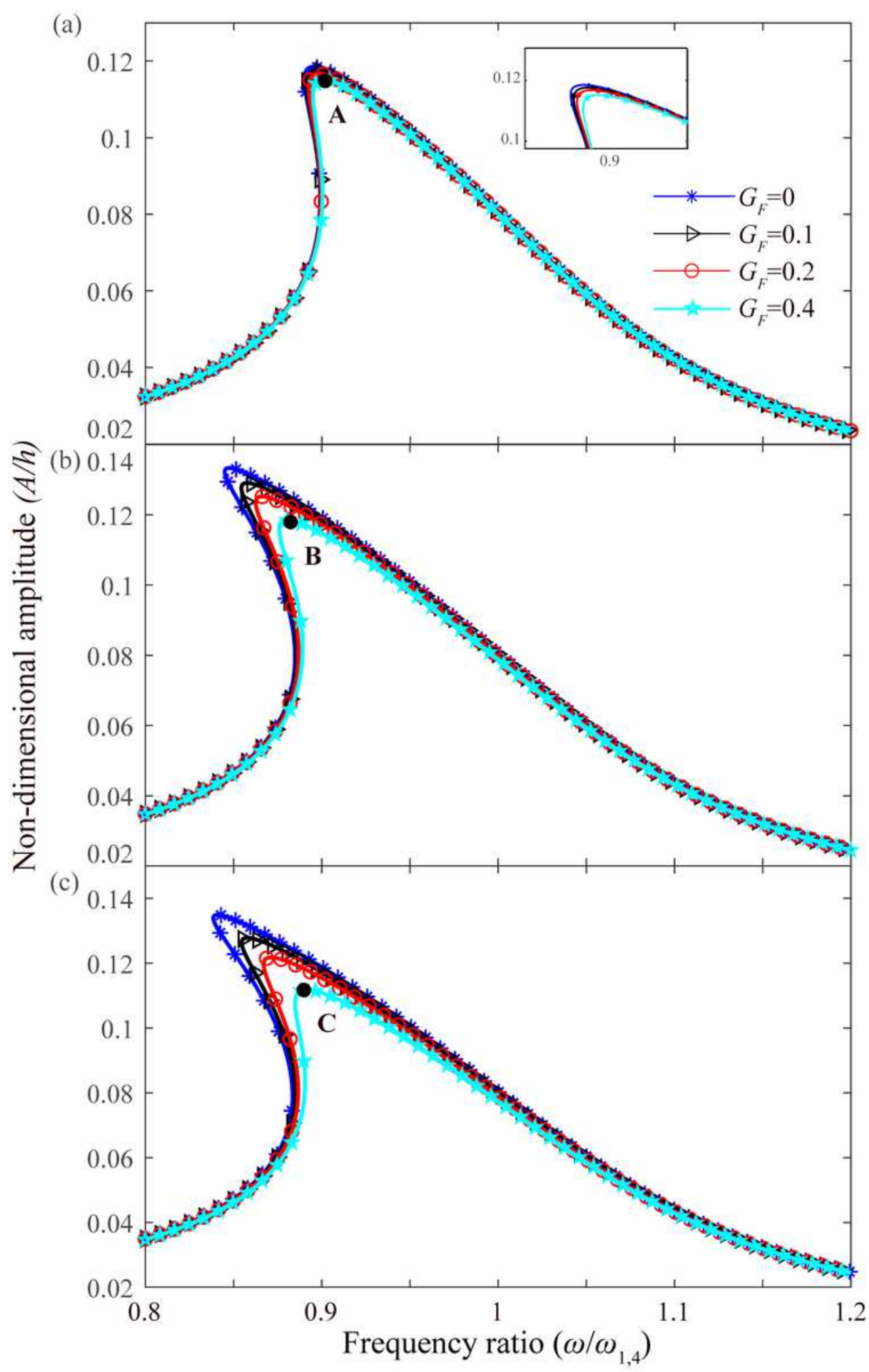

Figure 10

The influences of the position of the piezoelectric layer and constant gain for frequency-amplitude response 


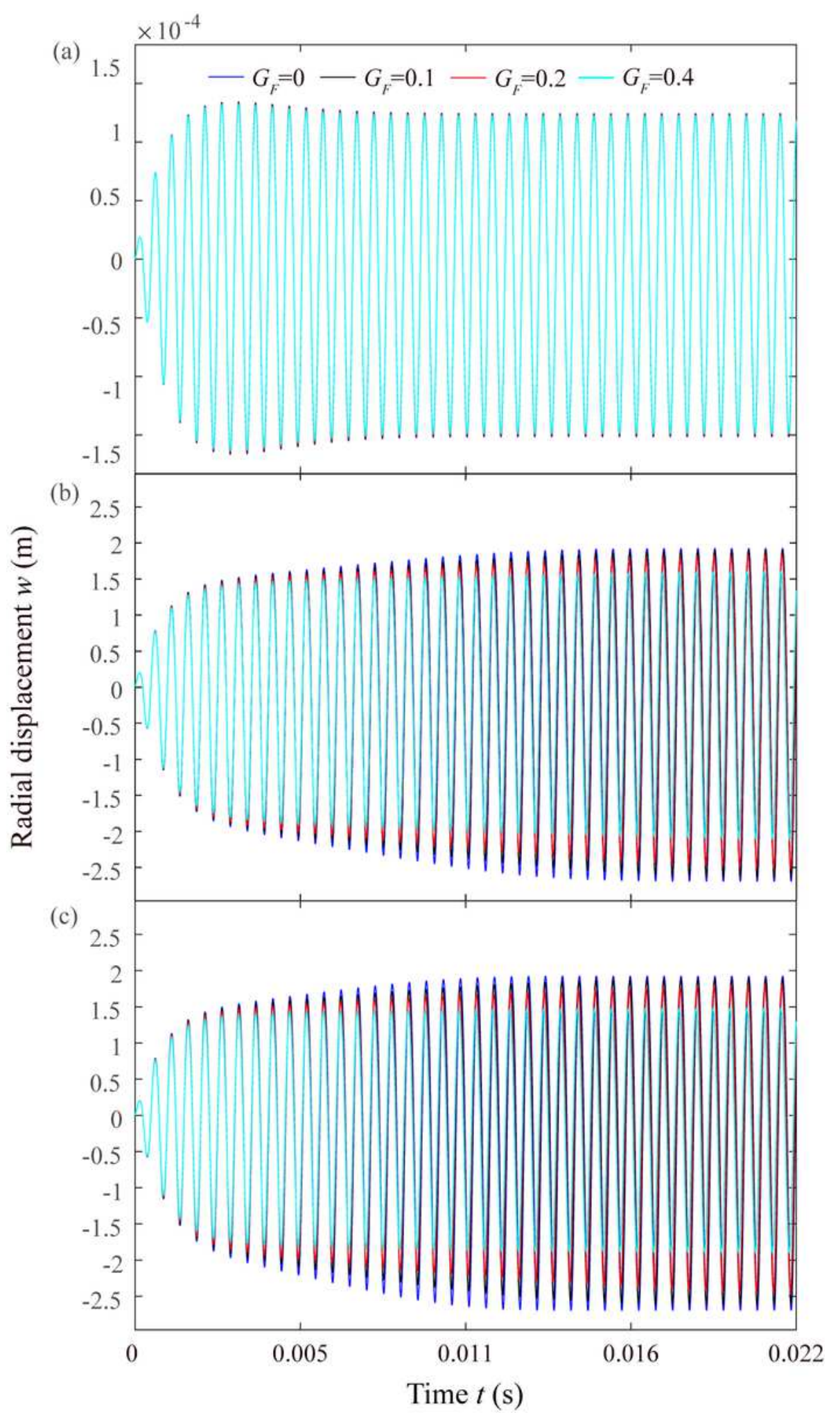

Figure 11

The influences of the position of the piezoelectric layer and constant gain for time-domain response 